\title{
A computable approach to measure and integration theory
}

\author{
Abbas Edalat* \\ Department of Computing, Imperial College, London SW7 2BZ, UK
}

\section{A R T I C L E I N F O}

\section{Article history:}

Received 20 March 2008

Available online 19 January 2009

\section{Keywords:}

Domain theory

Data type

Recursive and computable measurable set

Partial measurable set

Interval-valued measurable function

Interval-valued Lebesgue integral

\begin{abstract}
A B S T R A C T
We introduce a computable framework for Lebesgue's measure and integration theory in the spirit of domain theory. For an effectively given second countable locally compact Hausdorff space and an effectively given finite Borel measure on the space, we define a recursive measurable set, which extends the corresponding notion due to S̃anin for the Lebesgue measure on the real line. We also introduce the stronger notion of a computable measurable set, where a measurable set is approximated from inside and outside by sequences of closed and open subsets, respectively. The more refined property of computable measurable sets give rise to the idea of partial measurable subsets, which naturally form a domain for measurable subsets. We then introduce interval-valued measurable functions and develop the notion of recursive and computable measurable functions using intervalvalued simple functions. This leads us to the interval versions of the main results in classical measure theory. The Lebesgue integral is shown to be a continuous operator on the domain of interval-valued measurable functions and the interval-valued Lebesgue integral provides a computable framework for integration.
\end{abstract}

(c) 2009 Elsevier Inc. All rights reserved.

Double dedication: This paper is dedicated to the historical memory of Jamshid Kashani (d. 1429), the Iranian mathematician who was the first to use the recursive fixed point method in analysis with which he computed sin $1^{\circ} \operatorname{correct}$ to nine sexagesimal places; he is also well-known for computing $\pi$ to 16 decimal places [3, pp. 7 and 151]. This work is also dedicated to my colleague and friend Giuseppe Longo on the occasion of his 60th birthday to commend him for his wide range of interdisciplinary research interests and for his internationalist outlook on human culture.

\section{Introduction}

In the past decades, there has been a wide range of applications of measure and integration theory in different branches of computer science including in probabilistic semantics [24,18], stochastic hybrid systems [2] and labelled Markov processes $[11,7]$. Nevertheless, a systematic general framework for computability in measure and integration theory still remains in its infancy.

Computability of continuous functions and their integrals has been addressed by different schools in computable analysis (for example, [22, p. 37] and [27, p. 182]). In 1990's, the author developed a domain-theoretic framework for measure and integration theory which gave rise to a generalized Riemann integral $[8-10,12,19,1,20]$. It has provided a computable framework for measure theory and a generalised Riemann theory of integration. However, this theory only deals with almost everywhere continuous functions and not with measurable functions in general. Computability of measures on the unit interval has also been developed in type two theory [26] and, in addition, by using the Prokhorov distance in the metric space of measures [15].

\footnotetext{
* Fax: +61 283060405.

E-mail address: ae@doc.ic.ac.uk

URL: http://www.doc.ic.ac.uk/ ae/
} 
Computability of measurable subsets has a different story. In 1950's, based on the Russian approach to computability in analysis, Sanin [25] initiated research into computability of measurable sets in Euclidean spaces with respect to the Lebesgue measure. According to his definition, a bounded measurable set is recursive if there exists a recursive sequence of "simple" open sets, namely finite unions of bounded rational open intervals, such that the Lebesgue measure of the symmetric difference of the set and the elements of the sequence tends to zero effectively. This differs completely from Bishop's approach to constructive analysis [4].

The notion of a recursive measurable set is equivalent to that of a recursively approximable set, defined by Ker-I Ko in terms of a function-oracle [16]. The measure of the symmetric difference of two sets provides a pseudo-metric on the space of measurable subsets. S̃anin's notion is also at the basis of the approach adopted by researchers in type two theory of computability $[29,28]$, where an abstract computable measure space is defined as one which is generated by a countable ring of subsets and which is endowed with the pseudo-metric of the measure of symmetric difference.

We aim to develop here a new approach to computability of measurable sets and functions based on classical logic and recursion theory that is motivated by interval analysis and domain theory, where data types for mathematical objects are produced by providing lower and upper bounds for them. A directly relevant example is the computable framework for geometric objects in [13] in which a subset of a topological space is approximated from inside and outside by open subsets.

In this paper, we first develop an effective structure on any second countable, locally compact Hausdorff space and then derive the notion of an effectively given locally finite measure on such a space. We then extend recursive measurable sets to effectively given Borel measures on effectively given second countable compact Hausdorff spaces and show that they are closed under finite union, finite intersection and complementation. A recursive measurable set corresponds to a "rapidly converging" Cauchy sequence of basic open subsets with respect to the pseudo-metric of the measure of symmetric difference. Such a Cauchy sequence therefore provides a data type for measurable sets.

We then use a combined measure-theoretic and topological approach to define a computable measurable subset, which is given by the intersection of a recursive sequence of open sets containing the set and the union of a recursive sequence of closed sets contained in the set. Our notion of a computable measurable set, which gives approximations to a measurable set both from within and from outside, is stronger than S̃anin's recursive measurable sets. The contrast between the two notions can be seen in constructing elementary sets equivalent to a recursive and a computable measurable set. We will in fact construct a recursive $G_{\delta}$ set equivalent (up to a null set) to a given recursive measurable set on a compact space. For a computable measurable set, we can however construct an equivalent $F_{\sigma}$ set contained in it and an equivalent $G_{\delta}$ set containing it.

A computable measurable set in our framework is characterized for each positive integer $n$ by a recursively given closed set contained in the set and a recursively given open set containing the set, whose measure differ by less that $1 / 2^{n}$. This provides us with a more refined data type for measurable sets, namely measurable-set intervals or partial measurable sets, leading to a domain for measurable sets. In this domain, measurable sets are constructed as the least upper bound of increasing chains of basic partial measurable sets, each given by a pair of closed and open sets with the closed set contained in the open set. This is similar to the way the domain of real intervals, regarded as partial real numbers, forms a data-type for real numbers represented as the least upper bound of increasing chains of rational intervals.

Next we deal with measurable functions. We show generally that an interval-valued function on a measure space is measurable (with respect to the Borel $\sigma$-algebra induced by the Scott topology on the domain of intervals of the extended real line) if and only if the corresponding lower and upper extended real-valued functions are measurable. This observation allows us to develop measure theory for interval-valued maps on any measure space, giving rise to an $\omega$-bi-complete function space of measurable maps, which provides a domain for these maps.

Simple interval-valued measurable maps, which only take a finite number of interval values, are of particular interest: any bounded real-valued measurable function can be obtained as the supremum of an increasing chain of interval-valued simple measurable maps, which at each stage of computation gives lower and upper bounds for the real-valued function. Moreover, the chain can be chosen so that the simple function at level $n$ has, up to a constant factor independent of $n$, a total of $2^{n}$ distinct rational compact interval values each of width $2^{-n}$. This enables us to define a $\mu$-recursive and a $\mu$-computable measurable map and present a data type for such maps in terms of $\mu$-recursive and $\mu$-computable interval-valued simple maps, respectively.

The notion of $\mu$-computability enables us to develop a domain-theoretic data-type for measurable maps. A partial measurable set induces an interval-valued characteristic function, which is reduced to a simple interval-valued function in the domain of measurable maps. Moreover, in the domain of measurable maps, a $\mu$-computable measurable map can be constructed as the least upper bound of an effective increasing chain of simple interval-valued functions composed of characteristic maps of basic partial measurable sets. Therefore, we obtain a domain-theoretic data-type for $\mu$-computable measurable maps based on the domain of partial measurable sets.

As our main results, we derive the interval versions of the basic results in Lebesgue's theory of integration for bounded measurable functions with respect to finite Borel measures. In particular, the interval version of the monotone convergence theorem implies that the Lebesgue integral, as a functional on the space of interval-valued measurable functions, is $\omega$ continuous. This framework finally furnishes us with an effective method to compute the Lebesgue integral of a bounded computable measurable function with respect to an effectively given finite Borel measure on a second countable compact Hausdorff space.

It is assumed that the reader is familiar with the basic concepts of recursion theory as in [6], the elements of real number computability, in particular the notions of a computable real number and computable sequences of real numbers together 
with their main properties, as in [22, Chapter 0], and finally a basic knowledge of measure and integration theory as in $[23,17]$. For convenience, we denote both by $X \backslash A$ and by $A^{c}$ the complement of a subset $A \subseteq X$.

\section{Measurable sets}

In this section, we give two characterisations of measurable subsets with respect to a Borel measure on second countable locally compact Hausdorff spaces, which are used in the next section to define the notions of recursive measurable subsets and computable measurable subsets, respectively. We first recall a number of definitions; see [23].

A Hausdorff topological space is said to be $\sigma$-compact if there exists an increasing sequence of compact sets $\left(X_{i}\right)_{i \geqslant 0}$ with $X=\bigcup_{i \geqslant 0} X_{i}$. A measure $\mu$ on a topological space is said to be regular if for any $\mu$-measurable set $A$ we have:

$$
\mu(A)=\inf \{\mu(O): A \subseteq O, O \text { open }\}=\sup \{\mu(C): C \subseteq A, C \text { compact }\} .
$$

A Borel measure $\mu$ on a Hausdorff space $X$ is said to be locally finite if for any compact subset $K \subseteq X$ we have $\mu(K)<\infty$. We start by providing a simple characterization of measurable sets on locally compact second countable Hausdorff spaces. Assume for the rest of this section that $X$ is such a space.

Theorem 2.1. Let $X$ be a locally compact second countable Hausdorff space and $\mu$ a locally finite Borel measure on $X$. Then a subset $A \subseteq X$ is $\mu$-measurable iff for each $\epsilon>0$ there exists an open set $O$ and a closed set $C$ such that $C \subseteq A \subseteq O$ with $\mu(O \backslash C)<\epsilon$.

Proof. The "only if" part follows from Theorem 2.14 (Riesz's Representation Theorem) and Theorems 2.17 and 2.18 in [23] as follows. Since $X$ is second countable, every open set in $X$ is $\sigma$-compact, and it follows from Theorem 2.18 [23] that $\mu$ is regular. By Theorems 2.14 and 2.17 in [23], for the $\mu$-measurable subset $A$ and any $\epsilon>0$, there exist an open set $O$ and a closed set $C$ such that $C \subseteq A \subseteq O$ with $\mu(O \backslash C)<\epsilon$ as required. For the "if part", we put $\epsilon=1 / 2^{n}$ for any integer $n \geqslant 0$. Then there are open and closed sets $O_{n}$ and $C_{n}$ such that $C_{n} \subseteq A \subseteq O_{n}$ with $\mu\left(O_{n} \backslash C_{n}\right)<1 / 2^{n}$. Let $F=\cup_{n \geqslant 1} C_{n}$ and $G=\bigcap_{n \geqslant 1} O_{n}$. Then $F$ and $G$ are $F_{\sigma}$ and $G_{\delta}$ subsets, respectively, and we have $F \subseteq A \subseteq G$ with $\mu(G \backslash F)=0$. Hence, $A$ is $\mu$-measurable.

We will use the following consequence of the above theorem to develop our computability theory for measurable subsets.

Corollary 2.2. Let $X$ be a locally compact second countable Hausdorff space, with $X=\bigcup_{i \geqslant 0} X_{i}$ where each $X_{i}$ is compact, and $\mu$ a locally finite Borel measure on $X$. Then $A \subseteq X$ is $\mu$-measurable iff for each $i \geqslant 0$ and $\epsilon>0$, there are open sets $U_{i} \subseteq X$ and $V_{i} \subseteq X$ such that

$$
X_{i} \backslash U_{i} \subseteq A \cap X_{i} \subseteq V_{i} \cap X_{i}
$$

and

$$
\left.\mu\left(X_{i} \cap U_{i} \cap V_{i}\right)\right)<\epsilon .
$$

Proof. Suppose $A$ is $\mu$-measurable and $\epsilon>0$. Then by Theorem 2.1, there exist a closed set $C$ and an open set $O$ such that $C \subseteq A \subseteq O$ and $\mu(O \backslash C)<\epsilon$. Put $U_{i}=X \backslash C$ and $V_{i}=O$ for all $i \geqslant 0$. Conversely, if for each $i \geqslant 0$ and $\epsilon>0$ two open sets $U_{i}$ and $V_{i}$ with the above properties exist, then by Theorem 2.1, $X_{i} \cap A$ is $\mu$-measurable, and thus $A=\bigcup_{i \geqslant 0}\left(X_{i} \cap A\right)$ is also $\mu$-measurable.

Next, we give an alternative characterisation of measurable sets. Note that the limit superior of a sequence $\left(A_{n}\right)_{n \geqslant 0}$ of subsets of a set is defined as

$$
\limsup _{n \rightarrow \infty} A_{n}=\bigcap_{n \geqslant 0} \bigcup_{m \geqslant n} A_{m}
$$

Let $v$ be a measure on a measure space with $\sigma$-algebra $\mathscr{F}$ and let $A \Delta B$ denote the symmetric difference between subsets $A$ and $B$. The following distance, which gives a pseudo-metric on $\mathscr{F}$, is well known in measure theory [5].

Definition 2.3. The $d_{v}$-distance between $A_{1}, A_{2} \in \mathscr{F}$ is defined as $d_{v}\left(A_{1}, A_{2}\right)=v\left(A_{1} \Delta A_{2}\right)$.

The pseudo-metric $d_{v}$ is complete and there is a constructive witness for this completion property. We say that a Cauchy sequence $\left(s_{n}\right)_{n \geqslant 0}$ in a pseudo-metric space is rapidly converging if, for all $n \geqslant 0$, we have: $d\left(s_{i}, s_{j}\right)<1 / 2^{n}$ for all $i, j \geqslant n$; compare with the similar notion of a rapidly converging sequence of rational numbers in [27, p. 88].

Lemma 2.4. If $\left(A_{n}\right)_{n \geqslant 0}$ is a rapidly converging Cauchy sequence in $\left(\mathscr{F}, d_{v}\right)$, then it has $\lim _{\sup _{n \rightarrow \infty}} A_{n}$ as a limit. 
Proof. See [5, 1.12.6 Theorem(ii), p. 54].

Since any Cauchy sequence has a rapidly converging subsequence, it follows that every Cauchy sequence $\left(A_{n}\right)_{n \geqslant 0}$ in $\left(\mathscr{F}, d_{v}\right)$ has a limit of the form $\lim \sup _{n \rightarrow \infty} A_{i_{n}}$ for some subsequence $A_{i_{n}}$. This limit is therefore a limit of the original sequence as well.

Theorem 2.5. Given a Borel measure $\mu$ on a second countable locally compact Hausdorff space and a subset $A \subseteq X$, the following are equivalent:

(i) A is $\mu$-measurable,

(ii) A is equivalent (up to a nullset) to $a G_{\delta}$ subset.

(iii) For each $\epsilon>0$ there exists an open $O$ such that $\mu(A \Delta O) \leqslant \epsilon$.

Proof. The implications (i) $\Longleftrightarrow$ (ii) and the implication (i) $\Rightarrow$ (iii) follow from Theorem 2.1. For (iii) $\Rightarrow$ (ii), assume for each $\epsilon=1 / 2^{n}(n \geqslant 0)$ that the open subset $A_{n}$ satisfies $\mu\left(A \Delta A_{n}\right)<1 / 2^{n}$. Thus, $\left(A_{n+1}\right)_{n \geqslant 0}$ is a rapidly converging Cauchy sequence and by Lemma 2.4, we have $\mu\left(A \Delta \lim \sup _{n \rightarrow \infty} A_{n}\right)=0$. Therefore, $A$ is equivalent (up to a nullset) to the $G_{\delta}$ set $\bigcap_{n \geqslant 0}\left(\cup_{m \geqslant n} A_{m}\right)$.

\section{Recursive measurable sets}

In this section, we develop the notion of a recursive measurable set based on the results of the previous section. We need to use computable sequences of real numbers and recall the following definitions and basic results from [22].

\section{Definition 3.1}

(i) A sequence $\left(r_{k}\right)_{k \geqslant 0}$ of rational numbers is computable if there exist three total recursive functions $a, b, s: \mathbb{N} \rightarrow \mathbb{N}$ with $b(k) \neq 0$ for $k \geqslant 0$ such that for all $k \geqslant 0$ :

$$
r_{k}=(-1)^{s(k)} \frac{a(k)}{b(k)}
$$

(ii) Let $\left(x_{k}\right)_{k \geqslant 0}$ be a sequence of real numbers with $x_{k} \rightarrow x$ as $k \rightarrow \infty$. We say that $x_{k} \rightarrow x$ converges effectively in $k$ if there exists a total recursive function $e: \mathbb{N} \rightarrow \mathbb{N}$ such that for all $N$ :

$$
k \geqslant e(N) \text { implies }\left|x_{k}-x\right| \leqslant 2^{-N} .
$$

(iii) Let $\left(x_{n k}\right)_{n, k \geqslant 0}$ be a double sequence of real numbers with $x_{n k} \rightarrow x_{n}$ as $k \rightarrow \infty$ for each $n \geqslant 0$. We say that $x_{n k} \rightarrow x_{n}$ converges effectively in $n$ and $k$ as $k \rightarrow \infty$ if there exists a total recursive function $e: \mathbb{N} \times \mathbb{N} \rightarrow \mathbb{N}$ such that for all $n, N$ :

$$
k \geqslant e(n, N) \quad \text { implies } \quad\left|x_{n k}-x_{n}\right| \leqslant 2^{-N} .
$$

(iv) A real number $x$ is computable if there is a computable sequence of rational numbers $\left(r_{n}\right)_{n \geqslant 0}$ such that $r_{n} \rightarrow x$ effectively in $n$.

(v) A sequence of real numbers $\left(x_{n}\right)_{n \geqslant 0}$ is computable (as a sequence) if there is a computable double sequence of rationals $\left(r_{n k}\right)_{n, k \geqslant 0}$ such that $r_{n k} \rightarrow x_{n}$ as $k \rightarrow \infty$ effectively in $n$ and $k$.

(vi) A function $f: \mathbb{R} \rightarrow \mathbb{R}$ is computable if (1) it maps computable sequences of real numbers to computable sequences of real number, and (2) it is effectively uniformly continuous on intervals $[-n, n]$, i.e., there is a recursive function $h: \mathbb{N}^{2} \rightarrow \mathbb{N}$ such $|x-y| \leqslant 1 / 2^{h(n, k)}$ and $x, y \in[-n, n]$ implies $|f(x)-f(y)| \leqslant 1 / 2^{k}$ for all $n, k \in \mathbb{N}$ and $x, y \in \mathbb{R}$.

It is easy to extend (ii) to triple sequences of real numbers. Next, we need the following basic results about computable sequences of real numbers.

\section{Proposition 3.2}

(i) If $\left(a_{i j n}\right)_{i, j, n \geqslant 0}$ is a computable triple sequence of real numbers converging effectively in $i, j$ and $n$ as $j \rightarrow \infty$ with $\lim _{j \rightarrow \infty} a_{i j n}=b_{i n}$ then $\left(b_{i n}\right)_{i, n \geqslant 0}$ is a computable double sequence of real numbers.

(ii) If $\left(a_{i j}\right)_{i, j \geqslant 0}$ is a computable double sequence of real numbers which converges monotonically as $j \rightarrow \infty$ to a computable sequence of real numbers $\left(b_{i}\right)_{i \geqslant 0}$, i.e., $a_{i j} \leqslant a_{i(j+1)}$ for all $i, j \geqslant 0$ and $b_{i}=\lim _{j \rightarrow \infty} a_{i j}$ then the convergence of $\left(a_{i j}\right)_{i, j \geqslant 0}$ is effective in both $i$ and $j$ as $j \rightarrow \infty$.

Proof. (i) This is a straightforward extension of the result in [22, p. 20] for computable double sequences of real numbers to computable triple sequence of real numbers. 
(ii) See [22, p. 20].

We first develop the notions of recursive and computable measurable sets for a finite Borel measure on a compact second countable Hausdorff space $X$ which will highlight the basic ideas and results. In a later section, these ideas and results are extended to the technically more involved case of locally finite Borel measures on locally compact spaces. We start by presenting a notion of effective structure for a second countable compact Hausdorff space $X$ by equipping its $\omega$-continuous lattice of open subsets with an effective structure as a domain.

Definition 3.3. We say that a second countable compact Hausdorff space $X$ is effectively given with respect to an effective enumeration $\left(O_{i}\right)_{i \geqslant 0}$ of a countable basis of open sets closed under finite union and intersection if the following holds:

(i) $O_{0}=\emptyset$ and $O_{1}=X$.

(ii) There are total recursive functions $\phi$ and $\psi$ such that $O_{i} \cup O_{j}=O_{\phi(i, j)}$ and $O_{i} \cap O_{j}=O_{\psi(i, j)}$.

(iii) The predicates $O_{i} \subseteq O_{j}$ and $\overline{O_{i}} \subseteq O_{j}$ are decidable for $i, j \geqslant 0$.

Since $O_{i}=\emptyset$ iff $\overline{O_{i}} \subseteq O_{0}$, it follows that the equality relation $O_{i}=\emptyset$ is decidable and we can assume, by redefining the enumeration $\left(O_{i}\right)_{i \geqslant 0}$, that $O_{i}=\emptyset$ iff $i=0$. We note here that it would be possible to drop the requirement for the decidability of $O_{i} \subseteq O_{j}$ in (iii) at the expense of some more work. For simplicity though, we choose to keep this condition in our framework.

Proposition 3.4. For each $i \geqslant 0$, the predicate $X \backslash O_{i} \subseteq \bigcup_{1 \leqslant m \leqslant n} O_{i_{m}}$ is decidable for any finite set of integers $i_{m} \geqslant 0$ with $1 \leqslant$ $m \leqslant n$, i.e., from any effective covering of the compact subset $X \backslash O_{i}$ by basic open subsets, one can effectively obtain a finite subcovering.

Proof. Recall that $X=O_{1}$. Thus, the relation $X \backslash O_{i} \subseteq \bigcup_{1 \leqslant m \leqslant n} O_{i_{m}}$, in other words $X \subseteq O_{i} \cup\left(\bigcup_{1 \leqslant m \leqslant n} O_{i_{m}}\right)$, is equivalent, by using the total recursive function $\phi$ on the right hand side $n-1$ times, to $O_{1} \subseteq O_{j}$. The latter is decidable by condition (i) in Definition 3.3.

Now assume that $\mu$ is a finite Borel measure on the effectively given compact second countable space $X$ with its effective enumeration $\left(O_{i}\right)_{i \geqslant 0}$ of basic open sets.

Definition 3.5. The finite measure $\mu$ is effectively given on $X$ if $\left(\mu\left(O_{i}\right)\right)_{i \geqslant 0}$ is a computable sequence of real numbers.

We now use an effective version of the statement in Theorem 2.5 to define a recursive measurable set.

Definition 3.6. Suppose $\mu$ is effectively given on $X$. We say a measurable subset $A \subseteq X$ is a $\mu$-recursive measurable set if there exists a total recursive function $\lambda: \mathbb{N} \rightarrow \mathbb{N}$ such that $\mu\left(A \Delta O_{\lambda(n)}\right)<1 / 2^{n}$ for all $n \geqslant 0$.

By Theorem 2.5, we know that a $\mu$-recursive measurable set is indeed measurable (assuming that $A \Delta O_{\lambda(n)}$ is measurable for all $n \geqslant 0)$. Note that in the above definition, the Cauchy sequence $\left(O_{\lambda(n+1)}\right)_{n \geqslant 0}$ is rapidly converging with $A$ as a limit. We can therefore always assume that $\mu$-recursive measurable sets are defined by rapidly converging effective Cauchy sequences of basic open sets. It thus follows, from Lemma 2.4 , that there is a one to one correspondence between $\mu$-recursive measurable sets and rapidly converging effective Cauchy sequences of basic open sets, i.e. sequences of the form $\left(O_{\lambda(n)}\right)_{n \geqslant 0}$ for a total recursive function $\lambda$ satisfying, for all $n \geqslant 0$, the relation $\mu\left(O_{\lambda(i)} \Delta O_{\lambda(j)}\right)<1 / 2^{n}$ for all $i, j \geqslant n$.

Corollary 3.7. A $\mu$-recursive measurable set is up to a null set a $G_{\delta}$ set whose $\mu$-measure is a computable real number.

Proof. Suppose $A \subseteq X$ is a $\mu$-recursive measurable set with a total recursive function $\lambda: \mathbb{N} \rightarrow \mathbb{N}$ such that $\mu\left(A \Delta O_{\lambda(n)}\right)<1 / 2^{n}$ for all $n \geqslant 0$. By Theorem 2.5, we know that $A$ is equivalent to a $G_{\delta}$ set. On the other hand, since $\mu$ is an effectively given measure, $\left(\mu\left(O_{n}\right)\right)_{n \geqslant 0}$ is a computable sequence of real numbers and, therefore, so is $\mu\left(O_{\lambda(n)}\right)_{n \geqslant 0}$ as $\lambda$ is a total recursive function. The latter sequence converges effectively to $\mu(A)$, which is therefore a computable number. (Note that the limit of a computable sequence of real numbers which converges effectively is a computable real number [22, page 20]).

We also have the following closure properties of $\mu$-recursive measurable subsets.

Theorem 3.8. The complement, finite union and finite intersection of $\mu$-recursive measurable sets are $\mu$-recursive measurable sets.

Proof. Suppose $A$ and $B$ are $\mu$-recursive measurable subsets with total recursive functions $\lambda$ and $\eta$ satisfying:

$$
\mu\left(A \Delta O_{\lambda(n)}\right)<1 / 2^{n}, \quad \mu\left(B \Delta O_{\eta(n)}\right)<1 / 2^{n} .
$$


Note that $O_{\lambda(n)} \cup O_{\eta(n)}=O_{\phi(\lambda(n), \eta(n))}$ and for any subsets $C, C^{\prime}, D, D^{\prime}$ we have: $(C \cup D) \Delta\left(C^{\prime} \cup D^{\prime}\right) \subseteq\left(C \Delta C^{\prime}\right) \cup\left(D \Delta D^{\prime}\right)$. Thus,

$$
\mu\left((A \cup B) \Delta O_{\phi(\lambda(n), \eta(n))}\right)=\mu\left((A \cup B) \Delta\left(O_{\lambda(n)} \cup O_{\eta(n)}\right)\right)<\frac{1}{2^{n}}+\frac{1}{2^{n}}=\frac{1}{2^{n-1}},
$$

and hence the finite union of $\mu$-recursive measurable sets is $\mu$-recursive.

Next we show that the complement of $A$ is $\mu$-recursive. Consider the compact set $O_{\lambda(n+1)}^{c}$. Since the basis $\left(O_{i}\right)_{i \geqslant 0}$ is closed under finite unions and by Proposition 3.4, there is a total recursive function $\theta$ such that the subset relation $O_{\lambda(n+1)}^{c} \subseteq O_{\theta(i)}$ for $i \geqslant 0$ enumerates the union of all finite open covers of $O_{\lambda(n+1)}^{c}$ by basis elements. In fact, we can take $\theta(i)$ to be, in the usual ordering of natural numbers, the $i$ th natural number $j$ satisfying the relation $X=O_{1} \subseteq O_{\phi(j, \lambda(n+1))}$, a decidable predicate by Definition 3.3. We have $\bigcap_{i \geqslant 0} O_{\theta(i)}=O_{\lambda(n+1)}^{c}$, since for any point $x \in O$ one can find, by the Hausdorff property, a finite covering of $O_{\lambda(n+1)}^{c}$ by basic open sets that do not contain $x$. It follows that there exists $i \geqslant 0$ such that $\mu\left(O_{\theta(i)} \backslash O_{\lambda(n+1)}^{c}\right)=$ $\mu\left(O_{\theta(i)} \cap O_{\lambda(n+1)}\right)<1 / 2^{n+1}$, or in other words, there exists $i \geqslant 0$ with:

$$
\mu\left(O_{\psi(\theta(i), \lambda(n+1))}\right)<1 / 2^{n+1} .
$$

The above relation is semi-decidable as $\mu\left(O_{i}\right)$ is a computable real number for any $i \geqslant 0$. We compute, in parallel, increasingly accurate approximations to the computable numbers $\mu\left(O_{\psi(\theta(i), \lambda(n+1))}\right)$ for a finite but increasing number of $i \geqslant 0$. Let $i(n)$ be the first integer in this parallel computation scheme so that the corresponding approximation yields a result strictly less than $1 / 2^{n+1}$, i.e. for which the above inequality holds. Then,

$$
\begin{aligned}
\mu\left(A^{c} \Delta O_{\theta(i(n))}\right) & \leqslant \mu\left(A^{c} \Delta O_{\lambda(n+1)}^{c}\right)+\mu\left(O_{\lambda(n+1)}^{c} \Delta O_{\theta(i(n))}\right) \\
& =\mu\left(A \Delta O_{\lambda(n+1)}\right)+\mu\left(O_{\lambda(n+1)}^{c} \Delta O_{\theta(i(n))}\right) \\
& <\frac{1}{2^{n+1}}+\mu\left(O_{\psi(\theta(i(n)), \lambda(n+1))}\right) \\
& <\frac{1}{2^{n+1}}+\frac{1}{2^{n+1}}=\frac{1}{2^{n}} .
\end{aligned}
$$

This shows that $A^{c}$ is $\mu$-recursive. Since finite intersections of sets can be written as the complement of the union of their complements, the result follows.

For the Lebesgue measure $\mu$ on the real line, a countable union of $\mu$-recursive open sets may not be a $\mu$-recursive open set. In the next section, we give a simple counter example of this property for $\mu$-computable open sets which will also be a counter example for the case of $\mu$-recursive open sets.

\section{Computable measurable sets}

In this section, we introduce the notion of a computable measurable set for Borel measures on compact second countable Hausdorff spaces. Assume that $\mu$ is an effectively given finite Borel measure on the effectively given compact second countable space $X$ with its effective enumeration $\left(O_{i}\right)_{i \geqslant 0}$ of basic open sets. Recall from Theorem 2.1 that a subset $A \subseteq X$ is measurable iff for all $\epsilon>$ there exist a closed set $C$ and an open set $O$ with $C \subseteq A \subseteq O$ and $\mu(O \backslash C)<\epsilon$. Our task is to make this property effective with respect to the basic open subsets $\left(O_{i}\right)_{i \geqslant 0}$ and the corresponding closed subsets $\left(O_{i}^{C}\right)_{i \geqslant 0}$. From Proposition 3.4, it follows that there exists a total recursive function

$$
\alpha: \mathbb{N} \rightarrow \mathbb{N}^{2}
$$

such that $\vec{O}_{\alpha(j)}:=\left(O_{\alpha_{1}(j)}, O_{\alpha_{2}(j)}\right)$ gives an enumeration of covers of $X$ by pairs of basic open sets, i.e., $O_{\alpha_{1}(j)} \cup O_{\alpha_{2}(j)}=X$, or equivalently, $\left(O_{\alpha_{1}(j)}\right)^{c} \subseteq O_{\alpha_{2}(j)}$. This motivates the following formulation.

Definition 4.1. We say $A \subseteq X$ is a $\mu$-computable measurable set if there exists a total recursive function $\beta: \mathbb{N}^{2} \rightarrow \mathbb{N}$ such that the following holds:

(i) The two sequences $\left(O_{\alpha_{1}(\beta(j, n))}\right)_{j, n \geqslant 0}$ and $\left(O_{\alpha_{2}(\beta(j, n))}\right)_{j, n \geqslant 0}$ of open sets are increasing in $j \geqslant 0$ for fixed $n \geqslant 0$ and decreasing in $n$ for fixed $j$.

(ii) For all $n \geqslant 0$, we have: $\left(\bigcup_{j \geqslant 0} O_{\alpha_{1}(\beta(j, n))}\right)^{c} \subseteq A \subseteq\left(\bigcup_{j \geqslant 0} O_{\alpha_{2}(\beta(j, n))}\right)$.

(iii) The two computable double sequences of real numbers $\left(\mu\left(O_{\alpha_{1}(\beta(j, n))}\right)\right)_{j, n \geqslant 0}$ and $\left(\mu\left(O_{\alpha_{2}(\beta(j, n))}\right)\right)_{j, n \geqslant 0}$ converge effectively in $j$ and $n$ as $j \rightarrow \infty$.

(iv) For all $n \geqslant 0$, we have: $\mu\left(\left(\bigcup_{j \geqslant 0} O_{\alpha_{1}(\beta(j, n))}\right) \cap\left(\bigcup_{j \geqslant 0} O_{\alpha_{2}(\beta(j, n))}\right)\right)<1 / 2^{n}$.

Item (i) ensures that we only need to work with monotonic sequences of open subsets to characterize the $\mu$-computability of a measurable subset $A$. Items (ii) and (iv) provide us, for each integer $n \geqslant 0$, with a closed and an open subset, contained 
in and containing $A$, respectively, and effectively given in terms of the basic open subsets, such that the measure of their difference is $1 / 2^{n}$. Item (iii) ensures that the $\mu$-measure of $A$ is computable.

We know by Theorem 2.1 that the conditions in Definition 4.1 above imply that $A$ is $\mu$-measurable. Moreover, we have:

Proposition 4.2. If $A$ is a $\mu$-computable measurable set then $\mu(A)$ is a computable real number.

Proof. Since the convergence in (iii) above is effective in $j$ and $n$ as $j \rightarrow \infty$, it follows from Proposition 3.2 that $\mu(X \backslash$ $\left.\bigcup_{j \geqslant 0} O_{\alpha_{1}(\beta(j, n))}\right)$ and $\mu\left(\bigcup_{j \geqslant 0} O_{\alpha_{2}(\beta(j, n))}\right)$ are computable sequences of real numbers, with the first one increasing and the second one decreasing in $n$. From (ii) and (iv), it follows that the common limit of these two sequences, i.e., $\mu(A)$, is a computable real number.

Proposition 4.3. Let $O=\bigcup_{j \geqslant 0} O_{\gamma(j)}$ where $\gamma: \mathbb{N} \rightarrow \mathbb{N}$ is a total recursive function. Then $O$ is a $\mu$-computable open set iff $\mu(0)$ is a computable real number.

Proof. If $O$ is $\mu$-computable then by Proposition 4.2, $\mu(O)$ is a computable real number. Now, for the converse, assume $\mu(O)$ is a computable real number. It suffices to show that there is an effective decreasing sequence of basic open sets whose complements are contained within $O$ with the sequence of the $\mu$-measure of these complements tending to $\mu(O)$. Using the total recursive function $\phi$ (Definition 3.3(ii)), we can assume without loss of generality that $\gamma$ gives rise to an increasing sequence of basic open sets. From $\gamma$ we can effectively obtain, by [14, Proposition 3], a total recursive function $\delta: \mathbb{N} \rightarrow \mathbb{N}$ such that $O=\bigcup_{j \geqslant 0} O_{\delta(j)}$ and $\overline{O_{\delta j}} \subseteq O_{\delta(j+1)}$ for all $j \geqslant 0$. Since $\left(\mu\left(O_{\delta(j)}\right)\right)_{j \geqslant 0}$ increases monotonically to the computable real number $\mu(0)$, the convergence is effective in $j$. Since $X$ is an effectively given compact space with respect to the basis $\left(O_{i}\right)_{i \geqslant 0}$ and since $\overline{O_{\delta j}} \subseteq O_{\delta(j+1)}$ for all $j \geqslant 0$, we can effectively find a finite open covering $X \backslash O_{\delta(j+1)} \subseteq \bigcup_{1 \leqslant m \leqslant n} O_{i_{m}}$ with $O_{i_{m}} \cap O_{\delta(j)}=\emptyset$ for $1 \leqslant m \leqslant n$. Using the total recursive function $\phi$ for binary union (Definition 3.3), we obtain a total recursive function $\sigma$ such that $X \backslash O_{\delta(j+1)} \subseteq O_{\sigma(j)}:=\bigcup_{1 \leqslant m \leqslant n} O_{i_{m}}$. Moreover, by putting $\theta(0):=\sigma(0)$ and $O_{\theta(j+1)}:=O_{\sigma(j)} \cap O_{\theta(j)}=O_{\psi(\sigma(j), \theta(j))}$, we obtain a total recursive function $\theta$ which induces an effective decreasing sequence of basic open sets that shares the above properties of the sequence induced by $\sigma$. This completes the proof.

The above characterization of a $\mu$-computable open set coincides with that in [13].

Corollary 4.4. Let $O=\bigcup_{j \geqslant 0} O_{\gamma(j)}$ where $\gamma: \mathbb{N} \rightarrow \mathbb{N}$ is a total recursive function. Then $O$ is a $\mu$-recursive open set iff $\mu(O)$ is a computable real number.

Proof. The "if" part follows from Proposition 4.3, since a $\mu$-computable measurable set is $\mu$-recursive. The "only if" part follows from Corollary 3.7.

In view of Definition 4.1, consider any total recursive function $\beta: \mathbb{N}^{2} \rightarrow \mathbb{N}$ which satisfies the following three conditions:

(E1) the two sequences $\left(O_{\alpha_{1}(\beta(j, n))}\right)_{j, n \geqslant 0}$ and $\left(O_{\alpha_{2}(\beta(j, n))}\right)_{j, n \geqslant 0}$ of open sets are increasing in $j$ for fixed $n$ and decreasing in $n$ for fixed $j$,

(E2) the two computable double sequences of real numbers $\left(\mu\left(O_{\alpha_{1}(\beta(j, n))}\right)\right)_{j, n \geqslant 0}$ and $\left(\mu\left(O_{\alpha_{2}(\beta(i, j, n))}\right)\right)_{j, n \geqslant 0}$ converge effectively in $j$ and $n$ as $j \rightarrow \infty$,

(E3) for all $n \geqslant 0$, we have the relation: $\mu\left(\left(\bigcup_{j \geqslant 0} O_{\alpha_{1}(\beta(j, n))}\right)\right) \cap\left(\left(\bigcup_{j \geqslant 0} O_{\alpha_{2}(\beta(j, n))}\right)\right)<1 / 2^{n}$.

Such a recursive function $\beta$ characterizes an equivalence class of $\mu$-computable measurable sets which differ by a null set. Two canonical representatives of this class are the $G_{\delta}$ set $\bigcap_{n \geqslant 0} \bigcup_{j \geqslant 0} O_{\alpha_{2}(\beta(j, n))}$ and the $F_{\sigma}$ set $\left(\bigcap_{n \geqslant 0} \bigcup_{j \geqslant 0} O_{\alpha_{1}(\beta(j, n))}\right)^{c}$.

Moreover, the one parameter family of pairs of closed and open sets in Definition 4.1(ii), for $n \geqslant 0$, represent a data-type for any member $A$ of this equivalence class where $\left(\bigcup_{j \geqslant 0} O_{\alpha_{1}(\beta(j, n))}\right)^{c} \subseteq A \subseteq\left(\bigcup_{j \geqslant 0} O_{\alpha_{2}(\beta(j, n))}\right)$ and the measure of the open subset and the closed subset in each pair differ by at most $1 / 2^{n}$, as can be seen follows from Definition 4.1(iv).

\section{Proposition 4.5}

(i) The complement of a computable measurable set is another computable measurable set.

(ii) A finite union or intersection of computable measurable subsets is a computable measurable subset.

Proof. (i) Interchange 1 and 2 in the indices of $\alpha$ and $\beta$ in Definition 4.1.

(ii) This follows easily using the total recursive functions $\phi$ and $\psi$ for binary union and binary intersection of basic open sets. intersection.

We provide a simple example to show that $\mu$-computable (or $\mu$-recursive) measurable subsets are not closed under countable union or intersection. 
Example 4.6. Consider the Lebesgue measure $\mu$ on the real line and let $\left(r_{k}\right)_{k \geqslant 0}$ be an effective increasing sequence of positive rational numbers converging to a left-computable but non-computable real number $r \in \mathbb{R}$. (Such a sequence can be constructed from a recursively enumerable but non-recursive subset of natural numbers.) Then by Proposition 4.3, the open interval $(0, r)=\bigcup_{k \geqslant 0}\left(0, r_{k}\right)$ is not $\mu$-computable though for each $k \geqslant 0$ the open interval $\left(0, r_{k}\right)$ is $\mu$-computable.

\section{Domain of measurable subsets}

We now introduce a domain for $\mu$-measurable sets and show our notion of a $\mu$-computable measurable set actually provides a domain-theoretic data type. Consider a measure space $(X, \mathscr{S})$ with the underlying set $X$ and a $\sigma$-algebra $\mathscr{S}$ of subsets of $X$, regarded as measurable subsets. The domain $(\mathbf{M}(X, \mathscr{S})$, ᄃ) of measurable subsets of $X$ is defined as follows. An element of $\mathbf{M}(X, \mathscr{S})$ is given by a pair of subsets $A, B \in \mathscr{S}$ with $A \subseteq B$. The partial order is defined as $\left[A_{1}, B_{1}\right] \subseteq\left[A_{2}, B_{2}\right]$ if $A_{1} \subseteq A_{2}$ and $B_{1} \supseteq B_{2}$. Thus, $(\mathbf{M}(X, \mathscr{S})$, $\subseteq$ ) is $\omega$-bi-complete with

$$
\bigsqcup_{i \geqslant 0}\left[A_{i}, B_{i}\right]=\left[\bigcup_{i \geqslant 0} A_{i}, \bigcap_{i \geqslant 0} B_{i}\right], \quad \prod_{i \geqslant 0}\left[A_{i}, B_{i}\right]=\left[\bigcap_{i \geqslant 0} A_{i}, \bigcup_{i \geqslant 0} B_{i}\right],
$$

and the pair $[\emptyset, X]$ as the least element. We think of $[A, B]$ as a partial, or partially defined, measurable set that can be refined to any measurable set $C$ with $A \subseteq C \subseteq B$, in much the same way that a real interval $[a, b]$ is regarded as a partial real number that can be refined to any real number $c$ with $a \leqslant c \leqslant b$.

Any measure on $(X, \mathscr{S})$ extends to the domain of partial measurable subsets as an interval-valued map. Let $[0, \infty]$ be the one-point compactification of $(0, \infty)$ and $\mathbf{I}[0, \infty]$ be the set of non-empty compact intervals of $[0, \infty]$ ordered by reverse inclusion. A measure $\mu$ on $(X, \mathscr{S})$ induces a map $\mathbf{M}(\mu): \mathbf{M}(X, \mathscr{S}) \rightarrow \mathbf{I}[0, \infty]$, defined by $\mathbf{M}(\mu):[A, B] \mapsto[\mu(A), \mu(B)]$. It is easy to check that this map is $\omega$-bi-continuous, i.e., it is monotone and preserves the supremums and infimums of increasing and decreasing $\omega$-chains.

Assume now that $\mu$ is a finite measure on the measure space $(X, \mathscr{S})$. We say that a subset $\mathscr{D} \subseteq \mathbf{M}(X, \mathscr{S})$ is a $\mu$-basis for the domain $\left(\mathbf{M}(X)\right.$, ᄃ) if for any $C \in \mathscr{S}$ there exists an increasing chain $\left[A_{n}, B_{n}\right]_{n \geqslant 0}$ in $\mathscr{D}$ with its lub $[A, B]:=\left[\bigcup_{n \geqslant 0} A_{n}, \bigcap_{n \geqslant 0} B_{n}\right]$ satisfying $A \subseteq C \subseteq B$ and $\mu(B \backslash A)=0$. An effective sequence $\left(S_{n}\right)_{n \geqslant 0}$, such that $S_{n} \in \mathscr{S}$ for all $n \geqslant 0, S_{0}=\emptyset$ and $S_{1}=X$, is said to be an $\omega$-generator sequence for the $\mu$-basis $\mathscr{D}$ of $(X, \mathscr{S})$ if the following two conditions hold:

(G-1) For every element $(A, B) \in \mathscr{D}$ in the $\mu$-basis, there are increasing sequences $\left(S_{f(n)}\right)_{n \geqslant 0}$ and $\left(S_{g(n)}\right)_{n \geqslant 0}$ for total functions $f, g: \mathbb{N} \rightarrow \mathbb{N}$ such that $A^{c}=\bigcup_{n \geqslant 0} S_{f(n)}$ and $B=\bigcup_{n \geqslant 0} S_{g(n)}$.

(G-2) The sequence $\left(\mu\left(S_{n}\right)\right)_{n \geqslant 0}$ is a computable sequence of real numbers.

We say that the $\mu$-basis element $[A, B]$ is effectively given (with respect to the $\omega$-generator sequence) if the total functions $f$ and $g$ in (G-1) above are recursive. We finally say that an element $C \in \mathscr{S}$ is $\mu$-domain computable with respect to the $\omega$-generator sequence $\left(S_{n}\right)_{n \geqslant 0}$ of the $\mu$-basis $\mathscr{D}$ if it is contained in the lub of an increasing recursive chain of effectively given $\mu$-basis elements with the width of the $n$th element of the chain bounded by $1 / 2^{n}$, i.e., if there exist total recursive functions $f: \mathbb{N}^{2} \rightarrow \mathbb{N}$ and $g: \mathbb{N}^{2} \rightarrow \mathbb{N}$ such that:

(C-1) For all $n \geqslant 0$ we have: $\left(\bigcup_{j \geqslant 0} S_{f(j, n)}\right)^{c} \subseteq C \subseteq \bigcup_{j \geqslant 0} S_{g(j, n)}$.

(C-2) The chain $\left(\left[\left(\bigcup_{j \geqslant 0} S_{f(j, n)}\right)^{c}, \bigcup_{j \geqslant 0} S_{g(j, n)}\right]\right)_{n \geqslant 0}$ is increasing in $n \geqslant 0$ and the sequences $\left(\mu\left(\bigcup_{j \geqslant 0} S_{f(j, n)}\right)\right)_{n \geqslant 0}$ and $\mu\left(\bigcup_{j \geqslant 0} S_{g(j, n)}\right)_{n \geqslant 0}$ are computable sequences of real numbers.

(C-3) For all $n \geqslant 0$, we have: $\mu\left(\bigcup_{j \geqslant 0} S_{g(j, n)}\right)-\mu\left(\bigcup_{j \geqslant 0} S_{f(j, n)}\right)^{c}<1 / 2^{n}$.

Now we consider a finite measure $\mu$ on a second countable compact Hausdorff space $X$. By Theorem 2.1, the collection $\mathscr{D}$ of pairs $(C, O)$, with $C$ closed and $O$ open, forms a $\mu$-basis for the domain $(\mathbf{M}(X, \mathscr{S})$, ㄷ) where $\mathscr{S}$ is the set of $\mu$ measurable subsets of $X$. Next assume further that $X$ is effectively given with respect to an enumeration $\left(O_{i}\right)_{i \geqslant 0}$ of a countable basis and $\mu$ is effectively given with respect to this enumeration as in Definition 3.5. Then, $\left(O_{i}\right)_{i \geqslant 0}$ is an $\omega$-generator sequence for $\mathscr{D}$ as (G-1) and (G-2) are satisfied. Finally we obtain:

Proposition 5.1. A $\mu$-computable measurable subset $A \subseteq X$ with respect to the enumeration $\left(O_{i}\right)_{i \geqslant 0}$ of basic open subsets is a $\mu$-domain computable subset with respect to $\left(O_{i}\right)_{i \geqslant 0}$ as an $\omega$-generator sequence of the basis of pairs of closed and open sets.

Proof. Let $\alpha$ and $\beta$ be as in Definition 4.1 for the $\mu$-computable measurable subset $A \subseteq X$. Putting $f=\alpha_{1} \circ \beta$ and $g=\alpha_{2} \circ \beta$ we see that the conditions (C-1)-(C-3) are met since by Proposition $3.2 \mu\left(\bigcup_{j \geqslant 0} O_{\alpha_{1}(\beta(j, n))}\right)$ and $\mu\left(\bigcup_{j \geqslant 0} O_{\alpha_{2}(\beta(j, n))}\right)$ are computable sequences of real numbers.

In Section 7, we will see how partial measurable sets naturally give rise to interval-valued measurable functions, which in turn provide a domain-theoretic data-type for measurable functions. 


\section{Locally compact spaces}

We will show how to extend the results of the previous two sections to locally finite measures on locally compact spaces. We will treat the case of $\mu$-computable measurable sets in detail and briefly indicate how the results for $\mu$-recursive measurable sets can be generalized in this setting. Let $X$ be a second countable locally compact Hausdorff space. Let $\left(O_{j}\right)_{j} \geqslant 0$ be an effective enumeration of a basis of relatively compact open sets, which is closed under non-empty finite intersection and finite union. We note that $X$ is $\sigma$-compact, i.e., there exists an increasing sequence of compact sets $\left(X_{i}\right)_{i \geqslant 0}$ with $X=\bigcup_{i \geqslant 0} X_{i}$, e.g., we can take $X_{i}=\bigcup_{n \leqslant i} \overline{O_{n}}$. For each $i \geqslant 0$, the collection $\left(O_{j} \cap X_{i}\right)_{j \geqslant 0}$ is a countable basis of the relative topology for $X_{i}$.

Definition 6.1. We say that $X$ is effectively given with respect to $\left(X_{i}\right)_{i \geqslant 0}$ and $\left(O_{j}\right)_{j \geqslant 0}$ if the following holds:

(i) $O_{0}=\emptyset$.

(ii) The predicates $O_{i} \subseteq O_{j}$ and $\overline{O_{i}} \subseteq O_{j}$ are decidable for $i, j \geqslant 0$.

(iii) There are total recursive functions $\phi$ and $\psi$ such that $O_{i} \cup O_{j}=O_{\phi(i, j)}$ and $O_{i} \cap O_{j}=O_{\psi(i, j)}$.

(iv) For each $i, j, k \geqslant 0$, the predicate $X_{i} \backslash O_{j} \subseteq O_{k} \cap X_{i}$ is decidable.

As in the compact case, we can and will assume that $O_{i}=\emptyset$ iff $i=0$. Notice however that in contrast to the compact setting (Definition 3.3), in the locally compact case we have an additional axiom (iv), which for a compact space $X$ follows from the other axioms by stipulating that $X=O_{1}$ as shown in Proposition 3.4.

From our assumptions, it follows that there exists a total recursive function $\alpha: \mathbb{N}^{2} \rightarrow \mathbb{N}^{2}$ such that $\vec{O}_{\alpha(i, j)}:=\left(O_{\alpha_{1}(i, j)}, O_{\alpha_{2}(i, j)}\right)$ gives an enumeration of covers of $X_{i}$ by pairs of basic open sets, i.e., $X_{i} \subseteq O_{\alpha_{1}(i, j)} \cup O_{\alpha_{2}(i, j)}$.

Example 6.2. As an important example, consider the real line $\mathbb{R}$ equipped with its Euclidean topology; it is a second countable locally compact Hausdorff space. We put $X_{i}=[-i, i]$ for $i \in \mathbb{N}$. Consider any effective enumeration $\left(U_{j}\right)_{j} \geqslant 0$ of the canonical basis $\mathscr{B}$ of $\mathbb{R}$ consisting of the set of finite unions of rational open intervals and assume $U_{0}=\emptyset$. Then $\mathscr{B}$ is closed under finite intersections as well and it is easily seen by checking the clauses of Definition 6.1 that $\mathbb{R}$ is effectively given with respect to $\left(X_{i}\right)_{i \geqslant 0}$ and $\left(U_{j}\right)_{j} \geqslant 0$. Moreover, there exists an effective scheme to express each $U_{i}$ as the finite union of disjoint open rational intervals, i.e., there exists a total recursive function $\rho: \mathbb{N} \rightarrow \mathbb{N}^{*}$ where $\mathbb{N}^{*}$ is the set of finite sequences over $\mathbb{N}^{*}$ such that if $\rho(i)=\left(\rho(i)_{0}, \rho(i)_{1}, \cdots, \rho(i)_{n_{i}}\right)$, for some $n_{i} \in \mathbb{N}$, then $U_{i}=U_{\rho(i)_{0}} \cup U_{\rho(i)_{1}} \cup \cdots \cup U_{\rho(i)_{n_{i}}}$, where $U_{\rho(i)_{m}}$ are disjoint intervals for $0 \leqslant m \leqslant n_{i}$. Let $\left(C_{i}\right)_{i \geqslant 0}$ be an effective enumeration of the set of compact rational intervals of $\mathbb{R}$. Since the interior $C_{i}^{\circ}$ is, for each $i \geqslant 0$, a rational open (possibly empty) interval, we can effectively obtain $j \geqslant 0$ such that $C_{i}^{\circ}=U_{j}$. Thus, there exists a total recursive function $\tau: \mathbb{N} \rightarrow \mathbb{N}$ such that $C_{i}^{\circ}=U_{\tau(i)}$ for all $i \geqslant 0$. Finally, there is a partial recursive function $\kappa: \mathbb{N} \rightarrow \mathbb{N}$ such that $C_{\kappa(i)}^{\circ}=U_{i}$ whenever $U_{i}$ is an open interval.

We now give two other equivalent characterizations of computable functions of type $\mathbb{R} \rightarrow \mathbb{R}$ as defined in Definition 3.1.

Lemma 6.3. A continuous function $f: \mathbb{R} \rightarrow \mathbb{R}$ is computable iff the relation $f\left[C_{i}\right] \subset C_{j}^{\circ}$ is r.e. in $i, j$.

Proof. This follows from [14, Theorems 26 and 29].

Theorem 6.4. A continuous function $f: \mathbb{R} \rightarrow \mathbb{R}$ is computable iff the relation $\overline{U_{i}} \subseteq f^{-1}\left(U_{j}\right)$ is r.e. in $i, j$.

Proof. Suppose the relation $\overline{U_{i}} \subseteq f^{-1}\left(U_{j}\right)$ is r.e. in $i, j$. We have: $f\left[C_{i}\right] \subseteq C_{j}^{\circ}$ iff $C_{i} \subseteq f^{-1}\left(C_{j}^{\circ}\right)$ iff $\overline{U_{\tau(i)}} \subseteq f^{-1}\left(U_{\tau(j)}\right)$. Since $\tau$ is a total recursive function the latter relation is by assumption r.e. in $i, j$ and thus so is the relation $f\left[C_{i}\right] \subseteq C_{j}^{\circ}$.

Suppose on the other hand that $C_{i} \subseteq f^{-1}\left(C_{j}^{\circ}\right)$ is r.e. in $i, j$. Since

$$
\begin{aligned}
& \overline{U_{i}} \subseteq f^{-1}\left(U_{j}\right) \\
& \Longleftrightarrow \overline{U_{\rho(i)_{0}} \cup U_{\rho(i)_{1}} \cup \cdots \cup U_{\rho(i)_{i}}} \subseteq f^{-1}\left(\overline{\left(U_{\rho(j)_{0}} \cup U_{\rho(j)_{1}} \cup \cdots \cup U_{\rho(j) n_{j}}\right.}\right),
\end{aligned}
$$

it is sufficient to show that the relation $\overline{U_{i_{0}} \cup U_{i_{1}} \cup \cdots \cup U_{i_{m}}} \subseteq f^{-1}\left(U_{j_{0}} \cup U_{j_{1}} \cup \cdots \cup U_{j_{n}}\right)$, where all open subsets are assumed to be open intervals, is r.e. in $\left(\left(i_{0}, i_{1}, \ldots, i_{m}\right),\left(j_{0}, j_{1}, \ldots, j_{n}\right)\right) \in \mathbb{N}^{*} \times \mathbb{N}^{*}$. (Recall that $\mathbb{N}^{*}$ is in bijective correspondence with $\mathbb{N}$ under the mapping $\left\{k_{1}, k_{2}, \ldots, k_{n}\right\} \mapsto p_{1}^{k_{1}} p_{2}^{k_{2}} \ldots p_{n}^{k_{n}}$ where $k_{1}<k_{2}<\cdots<k_{n}$ and $p_{i}$ is the $i$ th prime number.) We can assume that open sets are open intervals by invoking the recursive function $\kappa$ introduced above, with $U_{i}=C_{\kappa(i)}^{\circ}$, which is defined for $i \in \mathbb{N}$ iff $U_{i}$ is an open interval. Fix $(m, n) \in \mathbb{N}^{2}$. We have $\overline{U_{i_{0}} \cup U_{i_{1}} \cup \cdots \cup U_{i_{m}}} \subseteq f^{-1}\left(U_{j_{0}} \cup U_{j_{1}} \cup \cdots \cup U_{j_{n}}\right)$ iff $\forall k(0 \leqslant k \leqslant$ $m) \exists l(0 \leqslant l \leqslant n) . f\left[\overline{U_{i_{k}}}\right] \subseteq U_{j_{l}}$ (since $f\left[\overline{U_{i_{k}}}\right]$ is a compact interval for each $k$ ) iff $\forall k(0 \leqslant k \leqslant m) \exists l(0 \leqslant l \leqslant n) . f\left[C_{\kappa\left(i_{k}\right)}\right] \subseteq C_{\kappa\left(j_{l}\right)}^{\circ}$. 
From this, we can deduce that the set

$$
\begin{aligned}
& E_{m n}:= \\
& \left\{\left(\left(i_{0}, \ldots, i_{m}\right),\left(j_{0}, \ldots, j_{n}\right)\right): \forall k(0 \leqslant k \leqslant m) \exists l(0 \leqslant l \leqslant n) . f\left[C_{\kappa\left(i_{k}\right)}\right] \subseteq C_{\kappa\left(j_{l}\right)}^{\circ}\right\}
\end{aligned}
$$

is r.e. In fact, for any pair $k, l \in \mathbb{N}$, with $0 \leqslant k \leqslant m$ and $0 \leqslant l \leqslant n$, the set

$$
E_{m n}^{k l}=\left\{\left(\left(i_{0}, i_{1}, \ldots, i_{m}\right),\left(j_{0}, j_{1}, \ldots, j_{n}\right)\right): f\left[C_{\kappa\left(i_{k}\right)}\right] \subseteq C_{\kappa\left(j_{l}\right)}^{\circ}\right\}
$$

is r.e. as follows. Since $E_{m n}=\bigcap_{0 \leqslant k \leqslant m} \bigcup_{0 \leqslant l \leqslant n} E_{m n}^{k l}$ and since any finite union and any finite intersection of r.e. sets are r.e., it follows that $E_{m n}$ is r.e. Finally, put

$$
E:=\left\{\left(\left(i_{0}, \ldots, i_{m}\right),\left(j_{0}, \ldots, j_{n}\right)\right): m, n \in \mathbb{N} \text { and } \forall k \exists l . f\left[C_{\kappa\left(i_{k}\right)}\right] \subseteq C_{\kappa\left(j_{l}\right)}^{\circ}\right\}
$$

Then, $E=\bigcup_{(m, n) \in \mathbb{N}^{2}} E_{m n}$ and is thus r.e. since any countable union of r.e. sets is r.e. [21, 5.9], and the proof is complete.

Using Theorem 6.4, we can generalize the notion of a computable function to real-valued continuous functions on a locally compact second countable Hausdorff space.

Definition 6.5. A continuous function $f: X \rightarrow \mathbb{R}$ on the effectively given locally compact second countable Hausdorff space $X$ with the enumeration $\left(O_{i}\right)_{i \geqslant 0}$ of its basis elements is computable if the relation $\overline{O_{i}} \subseteq f^{-1}\left(U_{j}\right)$ is r.e. in $i, j$.

Assume now that $\mu$ is a locally finite Borel measure on the effectively given locally compact second countable space $X$ with its effective enumeration $\left(O_{i}\right)_{i \geqslant 0}$ of basis. We say that $\mu$ is effectively given on $X$ if $\left(\mu\left(O_{j} \cap X_{i}\right)\right)_{i, j \geqslant 0}$ is a computable double sequence of real numbers.

Definition 6.6. We say $A \subseteq X$ is a $\mu$-recursive measurable set if there exists a total recursive function $\lambda: \mathbb{N}^{2} \rightarrow \mathbb{N}$ such that the double computable sequence of real numbers $\left(\mu\left(O_{\lambda(n, i)}\right)\right)_{n, i \geqslant 0}$ converges effectively in $n$ and $i$ as $n \rightarrow \infty$ with $\mu((A \cap$ $\left.\left.X_{i}\right) \Delta\left(O_{\lambda(n, i)} \cap X_{i}\right)\right)<1 / 2^{n}$ for all $n \geqslant 0$.

One can extend the proof of Theorem 3.8 to show that the family of $\mu$-recursive measurable sets are closed are finite unions, intersections and complementation. We will not present the details here and instead move on to define $\mu$-computable measurable sets on locally compact spaces for which we will provide the proofs of the extended results.

Definition 6.7. We say $A \subseteq X$ is a $\mu$-computable measurable set if there exists a total recursive function $\beta: \mathbb{N}^{3} \rightarrow \mathbb{N}$ such that

(i) The two triple sequences $\left(O_{\alpha_{1}(i, \beta(i, j, n))}\right)_{i j, n \geqslant 0}$ and $\left(O_{\alpha_{2}(i, \beta(i, j, n))}\right)_{i, j, n \geqslant 0}$ of open sets are both increasing in $i$ for fixed $j$ and $n$, increasing in $j$ for fixed $i$ and $n$ and decreasing in $n$ for fixed $i$ and $j$.

(ii) For all $i, n \geqslant 0$, we have:

$$
\left(X_{i} \backslash \bigcup_{j \geqslant 0} O_{\alpha_{1}(i, \beta(i, j, n))}\right) \subseteq X_{i} \cap A \subseteq\left(X_{i} \cap \bigcup_{j \geqslant 0} O_{\alpha_{2}(i, \beta(i, j, n))}\right) .
$$

(iii) The two computable triple sequences of real numbers

$$
\left(\mu\left(X_{i} \cap O_{\alpha_{1}(i, \beta(i, j, n))}\right)\right)_{i, j, n \geqslant 0}, \quad\left(\mu\left(X_{i} \cap O_{\alpha_{2}(i, \beta(i, j, n))}\right)\right)_{i, j, n \geqslant 0},
$$

converge effectively in $i, j$ and $n$ as $j \rightarrow \infty$.

(iv) For all $i, n \geqslant 0$, we have:

$$
\mu\left(X_{i} \cap\left(\bigcup_{j \geqslant 0} O_{\alpha_{1}(i, \beta(i, j, n))}\right) \cap\left(\bigcup_{j \geqslant 0} O_{\alpha_{2}(i, \beta(i, j, n))}\right)\right)<1 / 2^{n} .
$$

We know by Theorem 2.1 that the conditions in Definition 6.7 above imply that $A$ is $\mu$-measurable. Moreover, we have:

Proposition 6.8. If $A$ is a computable $\mu$-measurable set then $\left(\mu\left(A \cap X_{i}\right)\right)_{i \geqslant 0}$ is a computable sequence of real numbers. 
Proof. Since the convergence in Definition 6.7(iii) above is effective in $i, j$ and $n$ as $j \rightarrow \infty$, it follows from Proposition 3.2 that $\mu\left(X_{i} \backslash \bigcup_{j \geqslant 0} O_{\alpha_{1}(i, \beta(i, j, n))}\right)$ and $\mu\left(X_{i} \cap \bigcup_{j \geqslant 0} O_{\alpha_{2}(i, \beta(i, j, n))}\right)$ are computable double sequences of real numbers, with the first one increasing and the second one decreasing in $n$ for fixed $i$. From (iv) it follows that these two double sequences of real numbers converge effectively in $n$ and $i$ to $\mu\left(A \cap X_{i}\right)$ as $n \rightarrow \infty$. Thus, by Proposition 3.2, $\left(\mu\left(A \cap X_{i}\right)\right)_{i \geqslant 0}$ is a computable sequence of real numbers.

Proposition 4.3 can be extended to obtain:

Proposition 6.9. Let $O$ be a recursive union of basic open sets. Then $O$ is a $\mu$-computable open set iff $\left(\mu\left(X_{i} \cap 0\right)\right)_{i \geqslant 0}$ is a computable sequence of real numbers.

Proof. If $O$ is $\mu$-computable then by Proposition 6.8, $\left(\mu\left(X_{i} \cap 0\right)\right)_{i \geqslant 0}$ is a computable sequence of real numbers. Now, for the converse, let $O=\bigcup_{j \geqslant 0} O_{\gamma(j)}$ where the sequence of open sets is increasing and $\gamma: \mathbb{N} \rightarrow \mathbb{N}$ is a total recursive function and assume $\left(\mu\left(X_{i} \cap 0\right)\right)_{i \geqslant 0}$ is a computable sequence of real numbers. Since $\bigcup_{j \geqslant 0} O_{\gamma(j)}$ can be used as the first sequence of open sets in Definition 6.7, it suffices to construct the second sequence. As in the proof of Proposition 4.3, from $\gamma$ we can effectively obtain, by [14, Proposition 3], a total recursive function $\delta: \mathbb{N} \rightarrow \mathbb{N}$ such that $O=\bigcup_{j \geqslant 0} O_{\delta(j)}$ and $\overline{O_{\delta(j)}} \subseteq O_{\delta(j+1)}$ for all $j \geqslant 0$. Consider the computable double sequence of real numbers $\left(\mu\left(X_{i} \cap O_{\delta(j)}\right)_{i j} \geqslant 0\right.$. It monotonically converges to the computable sequence of real numbers $\left(\mu\left(X_{i} \cap 0\right)\right)_{i \geqslant 0}$. Thus, by Proposition 3.2, the convergence is effective in $i$ and $j$. Since $X$ is effectively locally compact with respect to the basis $\left(O_{j}\right)_{j \geqslant 0}$ and the sequence of compact subsets $\left(X_{i}\right)_{i \geqslant 0}$ and since $\overline{O_{\delta j}} \subseteq O_{\delta(j+1)}$ for all $j \geqslant 0$, we can effectively find a finite open covering $X_{i} \backslash O_{\delta(j+1)} \subseteq \bigcup_{1 \leqslant m \leqslant n} O_{t_{m}}$ with $O_{t_{m}} \cap O_{\delta(j)}=\emptyset$ for $1 \leqslant m \leqslant n$. Using the total recursive function $\phi$ for binary union (Definition 6.1), we obtain a total recursive function $\sigma$ such that $X_{i} \backslash O_{\delta(j+1)} \subseteq O_{\sigma(j)}:=\bigcup_{1 \leqslant m \leqslant n} O_{t_{m}}$. Moreover, by putting $\theta(0):=\sigma(0)$ and $O_{\theta(j+1)}:=O_{\sigma(j)} \cap O_{\theta(j)}=O_{\psi(\sigma(j), \theta(j))}$, for $j \geqslant 0$, we obtain a total recursive function $\theta$ which induces an effective decreasing sequence of basic open sets which shares the above properties of the sequence induced by $\sigma$. Since the construction is effective in $i$, this completes the proof.

The proof of Proposition 4.5 easily extends to locally finite measures on locally compact spaces:

\section{Proposition 6.10}

(i) The complement of a computable measurable set is another computable measurable set.

(ii) A finite union or intersection of computable measurable subsets is a computable measurable subset.

\section{Measurable functions}

Let $(X, \mathscr{S})$ be a measure space with the underlying set $X$ and a $\sigma$-algebra $\mathscr{S}$ of subsets of $X$. We work with such a general space first to develop the notion of interval-valued measurable functions, which we will motivate shortly. Later, in order to develop a computability theory for measurable functions, we assume that $X$ is a locally compact second countable Hausdorff space, equipped with its $\sigma$-algebra of measurable subsets induced by a Borel measure $\mu$ on $X$, i.e., $\mathscr{S}$ will be the set of all $\mu$-measurable subsets of $X$.

Given any topological space $Y$, we say that a function $f: X \rightarrow Y$ is measurable if $f^{-1}(B) \in \mathscr{S}$ for any Borel subset $B \subseteq Y$. Let $\overline{\mathbb{R}}$ be the extended real line, i.e., the two point compactification $[-\infty, \infty]$ of $\mathbb{R}$, where the basic open sets are of the form $(a, b),[-\infty, b)$ and $(a, \infty]$, with $a, b \in \mathbb{R}$. Let $\mathbf{I} \mathbb{R}$, respectively, $\mathbf{I} \overline{\mathbb{R}}$, be the domain of the non-empty compact intervals of the real line, respectively, of the extended real line, ordered by reverse inclusion and equipped with its $\sigma$-algebra of Borel subsets induced from the Scott topology.

In classical measure theory, measurable maps are built up from characteristic maps of measurable sets. It is thus natural to seek to define computable measurable maps by using characteristic maps of $\mu$-recursive or $\mu$-computable measurable subsets. We have seen in Section 5 that the domain of partial measurable subsets provides a data type to present $\mu$-computable measurable subsets. The notion of characteristic maps can be extended to partial measurable subsets as follow. Define:

$$
\begin{aligned}
\chi: \mathbf{M}(X, \mathscr{S}) & \rightarrow(X \rightarrow \mathbf{I} \mathbb{R}) \\
{[A, B] } & \mapsto \chi_{[A, B]}
\end{aligned}
$$

with

$$
\chi_{[A, B]}: X \mapsto\left\{\begin{array}{ccc}
1 & \text { if } & x \in A \\
0 & \text { if } & x \notin B \\
{[0,1]} & \text { if } & x \in B \backslash A
\end{array}\right.
$$

It is easily checked that $\chi$ is $\omega$-continuous, i.e., it is monotone and preserves the lubs of increasing $\omega$-chains. 
Consider the set $\left(X \rightarrow_{\mathrm{m}} \mathbf{I} \overline{\mathbb{R}}\right)$ of measurable functions $f: X \rightarrow \mathbf{I} \overline{\mathbb{R}}$ partially ordered pointwise, i.e., $f \sqsubseteq g$ if $f(x) \sqsubseteq g(x)$ for all $x \in X$. Each such function is determined by the extended real-valued lower and upper parts $f^{-}$and $f^{+}$of $f$ defined such that for each $x \in X$ we have $f(x)=\left[f^{-}(x), f^{+}(x)\right]$.

Proposition 7.1. We have $f \in\left(X \rightarrow_{\mathrm{m}} \mathbf{I} \overline{\mathbb{R}}\right)$ iff $f^{-}, f^{+}$are measurable as extended real-valued functions.

Proof. We note first that the Scott topology on $\mathbf{I} \overline{\mathbb{R}}$ has a countable basis consisting of subsets of the form $\square I=\{y: y \subseteq I\}$ where $I$ is any rational open interval, i.e., $I=(a, b)$ or $I=(a, \infty]$ or $I=[-\infty, b)$ for rational numbers $a, b \in \mathbb{R}$. Since the Borel $\sigma$-algebra on any topological space is generated by the open sets, $f$ will be measurable iff $f^{-1}(\square I) \in \mathscr{S}$ for all rational open intervals $I$. The result now follows from observing the following relations:

$$
\begin{aligned}
f^{-1}(\square(a, \infty]) & =\left(f^{-}\right)^{-1}(a, \infty] \\
f^{-1}(\square([-\infty, b)) & =\left(f^{+}\right)^{-1}[-\infty, b) \\
f^{-1}(\square(a, b)) & =\left(f^{-}\right)^{-1}(a, \infty] \cap\left(f^{+}\right)^{-1}[-\infty, b)
\end{aligned}
$$

In fact, if $f^{-}$and $f^{+}$are both measurable, then $f$ will be measurable since by the above relations $f^{-1}(\square I)$ is measurable for any rational interval $I$. On the other hand if $f$ is measurable then by the first two relations, $\left(f^{-}\right)^{-1}(a, \infty]$ and $\left(f^{+}\right)^{-1}[-\infty, b)$ are measurable for all rational numbers $a$ and $b$ and it will follow that $f^{-}$and $f^{+}$are both measurable.

It follows immediately that the function space $\left(X \rightarrow_{m} \mathbf{I} \overline{\mathbb{R}}\right)$ is closed under finite sums; moreover we have:

Proposition 7.2. If $\alpha \in \mathbf{I} \mathbb{R}$ and $f \in\left(X \rightarrow_{\mathrm{m}} \mathbf{I} \overline{\mathbb{R}}\right)$ then $\alpha f \in\left(X \rightarrow_{\mathrm{m}} \mathbf{I} \overline{\mathbb{R}}\right)$.

Proof. We have:

$$
\begin{aligned}
\alpha f & =\left[\alpha^{-}, \alpha^{+}\right]\left[f^{-}, f^{+}\right] \\
& =\left[\min \left(\alpha^{-} f^{-}, \alpha^{-} f^{+}, \alpha^{+} f^{-}, \alpha^{+} f^{+}\right), \max \left(\alpha^{-} f^{-}, \alpha^{-} f^{+}, \alpha^{+} f^{-}, \alpha^{+} f^{+}\right)\right] \\
& \in(X \rightarrow \mathrm{m} \mathbf{I} \mathbb{R}),
\end{aligned}
$$

since the min and max of measurable maps are measurable.

Corollary 7.3. If $\alpha_{i} \in \mathbf{I} \mathbb{R}$ and $f_{i} \in\left(X \rightarrow_{\mathrm{m}} \mathbf{I} \overline{\mathbb{R}}\right)$ for $1 \leqslant i \leqslant n$ then $\sum_{i=1}^{n} \alpha_{i} f_{i} \in\left(X \rightarrow_{\mathrm{m}} \mathbf{I} \overline{\mathbb{R}}\right)$.

Since the supremum, respectively, infimum, of an increasing, respectively, decreasing, sequence of real-valued measurable functions is measurable, the poset $\left(X \rightarrow_{\mathrm{m}} \mathbf{I} \overline{\mathbb{R}}\right)$ is $\omega$-bi-complete, i.e., the supremum (respectively, infimum) of any increasing (respectively, decreasing) sequence of interval-valued measurable functions is an interval-valued measurable function. Similarly, since the supremum (respectively, infimum) of any (finite or) countable set of measurable functions is measurable, it follows that $\left(X \rightarrow_{\mathrm{m}} \mathbf{I} \overline{\mathbb{R}}\right)$ is $\omega$-inf complete and bounded $\omega$-sup complete. In fact, suppose $\left(f_{i}\right)_{i \geqslant 0}$ is a countable sequence of elements in $\left(X \rightarrow_{\mathrm{m}} \mathbf{I} \overline{\mathbb{R}}\right)$. Then $\Pi_{i \geqslant 0} f_{i}=\lambda x$. $\Pi_{i \geqslant 0} f_{i}(x)$, where $\Pi_{i \geqslant 0} f_{i}(x)$ is the closure in $\overline{\mathbb{R}}$ of the convex hull of $\bigcup_{i \geqslant 0} f_{i}(x)$, which is compact since $\overline{\mathbb{R}}$ is compact. On the other hand, if $\left(f_{i}\right)_{i \geqslant 0}$ is a bounded sequence, then $\bigsqcup_{i \geqslant 0} f_{i}=\lambda x . \bigsqcup_{i \geqslant 0} f_{i}(x)$ since $\bigsqcup_{i \geqslant 0} f_{i}(x)$ is the intersection of non-empty compact intervals is therefore a non-empty and compact interval.

Given a sequence of intervals $x_{i} \in \mathbf{I} \overline{\mathbb{R}}, i \geqslant 0$ and $x \in \mathbf{I} \overline{\mathbb{R}}$, we write $\lim _{i \rightarrow \infty} x_{i}=x$ if $x^{-}=\lim _{i \rightarrow \infty} x_{i}^{-}$and $x^{+}=\lim _{i \rightarrow \infty} x_{i}^{+}$ both exist in $\overline{\mathbb{R}}$ with respect to its compact topology.

Furthermore, we introduce a limit operation on sequences in $\mathbf{I} \overline{\mathbb{R}}$ which we denote by lim$^{*}$ :

$$
\begin{array}{cccc}
\lim ^{*}: & (\overline{\mathbb{I}})^{\omega} & \rightarrow & \mathbb{I} \overline{\mathbb{R}} \\
& \left(x_{i}\right)_{i \geqslant 0} & \mapsto & {\left[\liminf _{i \rightarrow \infty} x_{i}^{-}, \limsup _{i \rightarrow \infty} x_{i}^{+}\right]}
\end{array}
$$

Note that $\lim _{i \rightarrow \infty}^{*} x_{i}$ for $x_{i} \in \mathbf{I} \overline{\mathbb{R}}$ is precisely the set of all limits of convergent sequences $\left(a_{i}\right)_{i \geqslant 0}$ with $a_{i} \in x_{i}$. Note also that lim ${ }^{*}$ is monotone but not continuous.

This induces a limit operation on sequences in $\left(X \rightarrow_{m} \mathbf{I} \overline{\mathbb{R}}\right)$ as follows:

$$
\begin{array}{ccc}
\lim ^{*}:\left(X \rightarrow_{\mathrm{m}} \mathbf{I} \overline{\mathbb{R}}\right)^{\omega} & \rightarrow & \left(X \rightarrow_{\mathrm{m}} \mathbf{I} \overline{\mathbb{R}}\right) \\
\left(f_{i}\right)_{i \geqslant 0} & \mapsto & {\left[\liminf _{i \rightarrow \infty} f_{i}^{-}, \lim \sup _{i \rightarrow \infty} f_{i}^{+}\right]}
\end{array}
$$

If $f^{-}=\lim _{i \rightarrow \infty} f_{i}^{-}$and $f^{+}=\lim _{i \rightarrow \infty} f_{i}^{+}$both exist then we write $\lim _{i \rightarrow \infty} f_{i}=f=\left[f^{-}, f^{+}\right]$. Clearly in this case $\lim _{i \rightarrow \infty} f_{i}=$ $\lim _{i \rightarrow \infty}^{*} f_{i}$.

From the definition of a characteristic map in Eq. (2), we immediately obtain: 
Proposition 7.4. For any partial measurable subset $[A, B]$ the characteristic map $\chi_{[A, B]}: X \rightarrow \mathbf{I} \mathbb{R}$ is measurable.

In analogy with simple functions in classical measure theory on the one hand and step functions in domain theory on the other hand, we define the following:

Definition 7.5. Let $\left[A_{i}, B_{i}\right] \subseteq X$ be partial measurable subsets of $X$ for $1 \leqslant i \leqslant n$ and let $\alpha_{i} \in \mathbf{I} \mathbb{R}$ be real intervals for $1 \leqslant i \leqslant n$. Then

$$
s=\sum_{i=1}^{n} \alpha_{i} \chi_{\left[A_{i}, B_{i}\right]}: X \rightarrow \mathbf{I} \mathbb{R}
$$

is called an interval-valued simple function.

Since $\chi_{[A, B]}=\chi_{A}+[0,1] \chi_{B \backslash A}$, the characteristic function of a partial measurable function can be expressed in terms of characteristic function of measurable functions with interval coefficients. Therefore, without loss of generality, we assume from now on that an interval-valued simple function has the form $s=\sum_{i=1}^{n} \alpha_{i} \chi_{A_{i}}: X \rightarrow \mathbf{I} \mathbb{R}$. From this, we see that $s=\left[s^{-}, s^{+}\right]$ where $s^{ \pm}: X \rightarrow \mathbb{R}$ with $s^{ \pm}=\sum_{i=1}^{n} \alpha^{ \pm} \chi_{A_{i}}$ are both measurable functions. It follows that $s$ is an interval-valued measurable function. Note also that we exclude extended real intervals from the definition of a simple function and that, as in the classical case, $s$ takes only a finite number of values and does not depend on the particular representation in terms of measurable sets $A_{i}$ 's and intervals $\alpha_{i}$ 's. There is indeed a canonical representation of $s$ for which the $\alpha_{i}$ 's are precisely the distinct non-zero values of $s$ and $A_{i}$ is precisely the set where $s$ takes value $\alpha_{i}$. We define the order o(s) of $s$ to be the number of distinct non-zero values of $s$. Using the canonical representation of $s$ we also define the width $w(s)$ of $s$ as the maximum length of the intervals $\alpha_{i}$, i.e., $w(s)=\max \left\{\alpha_{i}^{+}-\alpha_{i}^{-}: 1 \leqslant i \leqslant n\right\}$. Finally, for the canonical representation, we define the maximum absolute value of $s$ by $m(s)=\max \left\{\left|\alpha_{i}^{-}\right|,\left|\alpha_{i}^{+}\right|: 1 \leqslant i \leqslant n\right\}$. We say that $s$ is a rational interval-valued simple function if $\alpha_{i}$ is a compact rational interval for $1 \leqslant i \leqslant n$.

We say $f: X \rightarrow \mathbf{I} \mathbb{R}$ is bounded by a compact interval $K \in \mathbf{I} \mathbb{R}$, if for all $x \in X$ we have: $K \sqsubseteq f(x)$; we denote this by $K \sqsubseteq f$. We only deal with bounded measurable functions in this paper.

Let the real-valued measurable function $f: X \rightarrow \mathbb{R}$ be bounded so that $|f| \leqslant M$ for some $M \geqslant 0$. Let $m$ be the least nonnegative integer such that $M<2^{m}$. For a positive integer $n$ and any integer $k$ with $-2^{m+n+1}+2 \leqslant k \leqslant 2^{m+n+1}$ let

$$
A_{n k}=f^{-1}\left(\frac{k-2}{2^{n+1}}, \frac{k}{2^{n+1}}\right),
$$

which is measurable since $f$ is measurable and the dyadic interval $\left(\frac{k-2}{2^{n+1}}, \frac{k}{2^{n+1}}\right)$ is an open set. For a measurable $f$, let

$$
s_{n}=\sum_{k=-2^{(m+n+1)}+2}^{2^{(n+m+1)}}\left[\frac{k-2}{2^{n}}, \frac{k}{2^{n}}\right] \chi_{A_{n k}},
$$

then, we have $f=\bigsqcup_{n \geqslant 0} s_{n}$ with $w\left(s_{n}\right) \leqslant 1 / 2^{n}$ with $o\left(s_{n}\right) \leqslant 2^{n+m+2}$. We have therefore shown.

Proposition 7.6. Given a measure space $X$, every bounded real-valued measurable function $f: X \rightarrow \mathbb{R}$ is the supremum of an increasing sequence of rational interval-valued simple functions $s_{n}$ with $o\left(s_{n}\right) \leqslant c 2^{n}$, where $c$ is a positive constant independent of $n$, and $w\left(s_{n}\right) \leqslant 1 / 2^{n}$.

The above proposition can easily be extended to bounded interval-valued measurable functions of type $X \rightarrow \mathbf{I} \mathbb{R}$.

\subsection{Recursive and computable measurable functions}

An effective version of Proposition 7.6 provides us with the notion of a $\mu$-recursive and a $\mu$-computable real-valued measurable function and our data type for such functions. Let $X$ be an effectively given compact second countable Hausdorff space as in Section 2. We fix an effectively given Borel measure $\mu$ on $X$.

\section{Definition 7.7}

(i) A rational interval-valued simple function

$$
s=\sum_{i=1}^{k} \alpha_{i} \chi_{A_{i}}: X \rightarrow \mathbf{I} \mathbb{R}
$$

is $\mu$-computable ( $\mu$-recursive) if for $1 \leqslant i \leqslant k$, the subset $A_{i} \subseteq X$ is a $\mu$-computable ( $\mu$-recursive) measurable set for $1 \leqslant i \leqslant k$. 
(ii) A sequence $\left(s_{n}\right)_{n \geqslant 0}$ of $\mu$-computable ( $\mu$-recursive) interval-valued simple functions is effectively given if there is an effective procedure to obtain $s_{n}$.

(iii) We say that $f: X \rightarrow \mathbb{R}$ is a $\mu$-computable ( $\mu$-recursive) bounded measurable function if there is an effective increasing sequence of $\mu$-computable ( $\mu$-recursive) interval-valued simple functions $s_{n}: X \rightarrow \mathbf{I} \mathbb{R}$ with $f=\bigsqcup_{n \geqslant 0} s_{n}$ such that

- there is an effectively given non-negative integer $M \geqslant 0$ with $m\left(s_{n}\right) \leqslant M$ for all $n \geqslant 0$,

- $w\left(s_{n}\right) \leqslant 1 / 2^{n}$ for all $n \geqslant 0$, and,

- $o\left(s_{n}\right) \leqslant c 2^{n}$ for some effectively given positive constant $c$ independent of $n \geqslant 0$.

Two remarks regarding the notion of a $\mu$-computable ( $\mu$-recursive) simple function are in order.

(i) First, note that Definition 7.7(i) of a $\mu$-computable ( $\mu$-recursive) simple function is independent of the choice of the representative of $s$ as the complement and the finite union of $\mu$-computable ( $\mu$-recursive) measurable sets are both $\mu$-computable measurable sets.

(ii) We note that for a classical simple function $s=\sum_{i=1}^{k} a_{i} \chi_{A_{i}}: X \rightarrow \mathbb{R}$ with $a_{i} \in \mathbb{R}$ the two definitions in parts (i) and (ii) of Definition 7.7 are consistent. Indeed, if $A_{i}$ 's are $\mu$-computable ( $\mu$-recursive) measurable sets and $a_{i}$ 's are rational numbers, so that $s$ is a $\mu$-computable ( $\mu$-recursive) simple function according to Definition 7.7(i), then putting $s_{n}=s$ for all $n \geqslant 0$ we see that $s$ is $\mu$-computable ( $\mu$-recursive) as a measurable function in the sense of Definition 7.7(ii). On the other hand, suppose the simple function $s$ with canonical representation $s=\sum_{i=1}^{k} a_{i} \chi_{A_{i}}: X \rightarrow \mathbb{R}$ is a $\mu$-computable $(\mu$ recursive) measurable function in the sense of Definition 7.7(ii). Let $V=\{0\} \cup\left\{a_{i}: 1 \leqslant i \leqslant k\right\}$ and put $r=\min \{|v-w|$ : $v, w \in V$ with $v \neq w$ \}. By assumption, there is an increasing sequence of $\mu$-computable ( $\mu$-recursive) simple functions $s_{n}$ with $s=\bigsqcup_{n} s_{n}$ and $w\left(s_{n}\right) \leqslant 1 / 2^{n}$. Fix $i$ with $1 \leqslant i \leqslant k$, and let $n$ be such that $1 / 2^{n}<r / 2$. Assume $s_{n}=\sum_{t=1}^{m_{n}} \beta_{n t} \chi_{B_{n t}}$. Then, for $\leqslant i \leqslant k$, we have

$$
A_{i}=\bigcup_{a_{i} \in \beta_{n t}} B_{n t}
$$

and it follows that $A_{i}$ is the finite union of $\mu$-computable ( $\mu$-recursive) measurable subsets and is thus a $\mu$-computable ( $\mu$-recursive) measurable subset by Theorem 3.8 and Proposition 4.5 .

Proposition 7.8. The set of bounded real-valued $\mu$-computable ( $\mu$-recursive) measurable functions is closed under multiplication by a computable real number and under taking sums, absolute value, maximum and minimum.

Proof. Suppose $f=\bigsqcup_{n \geqslant 0} s_{n}$ and $f^{\prime}=\bigsqcup_{n \geqslant 0} s_{n}^{\prime}$, where $s_{n}$ and $s_{n}^{\prime}$ are $\mu$-computable ( $\mu$-recursive) simple functions satisfying the conditions in Definition 7.7(ii) and in particular: $w\left(s_{n}\right) \leqslant 2^{n}, o\left(s_{n}\right) \leqslant c 2^{n}$ and $w\left(s_{n}^{\prime}\right) \leqslant 2^{n}, o\left(s_{n}^{\prime}\right) \leqslant c^{\prime} 2^{n}$. Assume $a \in \mathbb{R}$ be a computable real number; we will show that af is a $\mu$-computable ( $\mu$-recursive) measurable function. Let $M$ be effectively given with $s_{n} \leqslant M$ for all $n \geqslant 0$. Let the positive integer $p$ be such that $|a|<2^{p}-1$. From the computability of $a$, Definition 3.1, it follows that there is a sequence $\left(\gamma_{n}\right)_{n \geqslant 0}$ of compact rational intervals with $w\left(\gamma_{n}\right) \leqslant 1 / 2^{n}$ that has $a$ as their intersections. Note that if $s=\Sigma_{i=1}^{k} \alpha_{i} \chi_{A_{i}}: X \rightarrow \mathbb{I} \mathbb{R}$ is the canonical representation of any interval-valued simple function (i.e., with disjoint $A_{i}$ 's, equivalently distinct $\alpha_{i}$ 's) and if $\gamma$ is any compact interval, then $\gamma s=\Sigma_{i=1}^{k} \gamma \alpha_{i} \chi_{A_{i}}$, where $\gamma \alpha=\{x y: x \in \gamma, y \in \alpha\}$. We also have: $w(\alpha S) \leqslant \max \left(\left|\gamma^{-}\right|,\left|\gamma^{+}\right|\right) w(S)$ and $o(\alpha S) \leqslant o(s)$. Consider now $t_{n}:=\gamma_{n} s_{n}$. Clearly $t_{n}$ is $\mu$-computable ( $\mu$-recursive). We have $a f=\bigsqcup_{n \geqslant 0} t_{n}$, with $w\left(t_{n}\right) \leqslant 2^{p-n}, o\left(t_{n}\right) \leqslant o\left(s_{n}\right) \leqslant c 2^{n}$ and $m\left(t_{n}\right) \leqslant 2^{p} M$ for all $n \geqslant 0$. Since $p$ is independent of $n$, it follows easily that af is $\mu$-computable ( $\mu$-recursive). Next, we consider the other operations. In all these cases, we construct a new effective sequence of $\mu$-computable ( $\mu$-recursive) interval-valued simple functions. For the sum operation, we have: $f+f^{\prime}=\bigsqcup_{n \geqslant 0} s_{n}+s_{n}^{\prime}$. Note that for real intervals $\alpha, \beta \in \mathbf{I} \mathbb{R}$ and for subsets $A, B \subseteq X$ we have:

$$
\alpha \chi_{A}+\beta \chi_{B}=(\alpha+\beta) \chi_{A \cap B}+\alpha \chi_{A \backslash B}+\beta \chi_{B \backslash A} .
$$

Since $\mu$-computable ( $\mu$-recursive) measurable subsets are closed under finite union, finite intersection and complementation, it follows that for each $n \geqslant 0$, the interval-valued simple function $s_{n}+s_{n}^{\prime}$ is $\mu$-computable ( $\mu$-recursive). From Eq. 5, it also follows that $w\left(s_{n}+s_{n}^{\prime}\right) \leqslant 1 / 2^{n-1}$ and, since $o\left(s_{n}\right) \leqslant c 2^{n}$ and $o\left(s_{n}^{\prime}\right) \leqslant c^{\prime} 2^{n}$, then $o\left(s_{n}+s_{n}^{\prime}\right) \leqslant 3 k 2^{n}$ where $k=\max \left(c, c^{\prime}\right)$. Furthermore, $m\left(s_{n}+s_{n}^{\prime}\right) \leqslant m\left(s_{n}\right)+m\left(s_{n}^{\prime}\right)$. We conclude that $f+f^{\prime}$ is a $\mu$-computable ( $\mu$-recursive) measurable function. Next, we show that $|f|$ is $\mu$-computable ( $\mu$-recursive). We use the pointwise extension of the absolute value function to real intervals. Then, for any interval-valued simple function $s=\sum_{i=1}^{k} a_{i} \chi_{A_{i}}: X \rightarrow \mathbb{R}$ we have the interval-valued simple function: $|S|=\sum_{i=1}^{k}\left|\alpha_{i}\right| \chi_{A_{i}}$ with $w(|S|) \leqslant w(S), o(|S|) \leqslant o(s)$ and $m(|S|)=m(S)$. It follows that $|f|=\bigsqcup_{n \geqslant 0}\left|S_{n}\right|$ is a $\mu$-computable $(\mu-$ recursive) measurable function. Finally, we note that $\min \left(f, f^{\prime}\right)=\left(f+f^{\prime}-\left|f-f^{\prime}\right|\right) / 2$ and $\max \left(f, f^{\prime}\right)=\left(f+f^{\prime}+\left|f-f^{\prime}\right|\right) / 2$, from which we conclude that $\min \left(f, f^{\prime}\right)$ and $\max \left(f, f^{\prime}\right)$ are $\mu$-computable ( $\mu$-recursive) measurable functions.

Consider the increasing sequence of bounded $\mu$-computable measurable functions $\left(f_{k}\right)_{k \geqslant 0}$ with $f_{k}=\chi_{\left(0, r_{k}\right)}$, where $\left(r_{k}\right)_{k \geqslant 0}$ is the sequence of rational numbers in Example 4.6. Since $\sup _{k \geqslant 0} f_{k}=\chi_{(0, r)}$, we see that the supremum of a countable set of bounded $\mu$-computable ( $\mu$-recursive) measurable functions is not necessarily $\mu$-computable ( $\mu$-recursive). 
Given a $\mu$-computable simple function $s=\sum_{i=1}^{k} \alpha_{i} \chi_{A_{i}}$ as in Definition 7.7, it follows by the $\mu$-computability of the measurable subsets $A_{i}$ (for $1 \leqslant i \leqslant k$ ) that there are total recursive functions $\beta_{i}: \mathbb{N}^{2} \rightarrow \mathbb{N}$ such that for $1 \leqslant i \leqslant k$ we have (up to a null set):

$$
A_{i}=\bigsqcup_{n \geqslant 0}\left[C_{i n}, O_{i n}\right]
$$

where $C_{i n}=\left(\bigcup_{j \geqslant 0} O_{\alpha_{1}\left(\beta_{i}(j, n)\right)}\right)^{c}$ and $O_{i n}=\bigcup_{j \geqslant 0} O_{\alpha_{2}\left(\beta_{i}(j, n)\right)}$. Thus, we have $s=\bigsqcup_{n \geqslant 0} s_{n}$ with

$$
s_{n}=\sum_{i=1}^{k} \alpha_{i} \chi_{\left[C_{i n}, O_{i n}\right]} .
$$

We have thus shown.

Proposition 7.9. A $\mu$-computable simple function is the lub of an increasing recursive chain of simple functions made up of effectively given $\mu$-basis elements.

Finally, we present a condition for a computable continuous function as introduced in Definition 6.5 to be a $\mu$-computable map. We say a real-valued continuous function $f: X \rightarrow \mathbb{R}$ is effectively bounded if a nonnegative number $M$ is effectively given such that $|f(x)| \leqslant M$ for all $x \in X$.

Theorem 7.10. An effectively bounded, computable continuous real-valued function on an effectively given compact second countable Hausdorff space is $\mu$-computable with respect to an effectively given finite Borel measure $\mu$ on the space if the $\mu$-measure of the inverse image of each open dyadic rational interval is a computable real number.

Proof. Let $f: X \rightarrow \mathbb{R}$ be an effectively bounded, computable continuous real-valued function on an effectively given compact second countable Hausdorff space $X$ with enumeration $\left(O_{i}\right)_{i \geqslant 0}$ and let $\mu$ be an effectively given finite Borel measure on $X$. Let $M$ be the effectively given bound for $f$ and let $m, n, k$ and $A_{n k}$ be as in Eq. 3. We have the simple functions $s_{n}$ in Eq. 4 with $f=\bigsqcup_{n \geqslant 0} s_{n}$. Given the open interval $\left(\frac{k-2}{2^{n+1}}, \frac{k}{2^{n+1}}\right)$, with $-2^{m+n+1}+2 \leqslant k \leqslant 2^{m+n+1}$, there is $j \geqslant 0$ such that $U_{j}=\left(\frac{k-2}{2^{n+1}}, \frac{k}{2^{n+1}}\right)$ as defined in Example 6.2 and thus $A_{n k}=f^{-1}\left(U_{j}\right)$. Since, by computability of $f$, the relation $\overline{O_{i}} \subseteq f^{-1}\left(U_{j}\right)$ is, for fixed $j$, r.e. in $i$, it follows that there exists a total recursive function $\gamma: \mathbb{N} \rightarrow \mathbb{N}$ such that $A_{n k}=\bigcup_{j \geqslant 0} O_{\gamma(j)}$. By assumption, $\mu\left(A_{n k}\right)$ is a computable real number. Thus, by Proposition $4.3, A_{n k}$ is a $\mu$-computable measurable subset and it follows that $s_{n}$ is an effective sequence of $\mu$-computable interval-valued simple functions with lub $f$, which completes the proof.

\section{Interval Lebesgue integral}

We are now in a position to define the notion of interval Lebesgue integral as a map $\int:\left(X \rightarrow_{m} \mathbf{I} \overline{\mathbb{R}}\right) \rightarrow \overline{\mathbb{R}}$ with respect to a measure $\mu$ on the measure space $(X, \mathscr{S})$. Later in this section, in order to develop a computability theory, we work with a finite Borel measure $\mu$ on a locally compact second countable Hausdorff space $X$.

For a simple function $s \in\left(X \rightarrow_{\mathrm{m}} \mathbf{I} \overline{\mathbb{R}}\right)$ with a representative $s=\sum_{i=1}^{n} \alpha_{i} \chi_{A_{i}}: X \rightarrow \mathbf{I} \mathbb{R}$, which vanishes outside a set of finite measure, we define the $\mu$-integral of $s$ as:

$$
\int_{X} s d \mu=\sum_{i=1}^{n} \alpha_{i} \mu\left(A_{i}\right)
$$

It follows that $\int_{X} s d \mu=\left[\int_{X} s^{-} d \mu, \int_{X} s^{+} d \mu\right]$. Thus, as in the classical case, the integral of a simple function is independent of its representative. If $E \subseteq X$ is measurable, then $s \cdot \chi_{E}=\sum_{i=1}^{n} \alpha_{i} \chi_{A_{i} \cap E}$ is also a simple function and, as in the classical case, we define:

$$
\int_{E} s d \mu=\int_{X} s \cdot \chi_{E} d \mu
$$

We also immediately deduce the following.

Proposition 8.1. If $s$ and $t$ are simple interval-valued functions then, for compact intervals $a, b \in \mathbf{I} \mathbb{R}$ :

(i) $\int(a s+b t) d \mu=a \int s d \mu+b \int t d \mu$.

(ii) If $s \sqsubseteq t$ holds a.e., then $\int s d \mu \sqsubseteq \int t d \mu$. 
Now we deal with bounded measurable functions. We first consider a bounded measurable function $f \in(X \rightarrow \mathrm{m} \overline{\mathbf{I}})$ and define:

Definition 8.2. The Lebesgue integral of any bounded interval-valued measurable function $f$ on a measurable subset $E$ with respect to a bounded measure $\mu$ on $X$ is defined as:

$$
\int_{E} f d \mu=\bigsqcup\left\{\int_{E} s d \mu: \text { simple } s \sqsubseteq f\right\} .
$$

We immediately obtain the following formula for computing the interval-valued Lebesgue integral:

Proposition 8.3. $\int_{E} f d \mu=\left[\int_{E} f^{-} d \mu, \int_{E} f^{+} d \mu\right]$.

We usually write $\int f d \mu$ for $\int_{X} f d \mu$. The following results easily follow as in the classical case.

Proposition 8.4. If $f$ and $g$ are bounded measurable interval-valued functions and $a$ and $b$ are real numbers, then:

(i) $\int(a f+b g) d \mu=a \int f d \mu+b \int g d \mu$.

(ii) If $f \sqsubseteq g$ holds a.e., then $\int f d \mu \sqsubseteq \int g d \mu$.

(iii) If $A$ and $B$ are disjoint measurable subsets then

$$
\int_{A \cup B} f d \mu=\int_{A} f d \mu+\int_{B} f d \mu
$$

Note that, in Proposition 8.4(i) above, the linearity of the integral operator on interval-valued functions only holds for real coefficients whereas for simple functions this linearity extends to compact real intervals as in Proposition 8.1(i).

We can now obtain in a straightforward way, using Proposition 8.3, the interval version of some of the classical results in measure theory. Recall the definitions of $\lim _{\text {and }} \lim ^{*}$ in $\left(X \rightarrow_{m} \mathbf{I} \bar{R}\right)$.

Proposition 8.5 (Bounded Convergence Theorem). Let $f_{n} \in\left(X \rightarrow_{m} \mathbf{I} \overline{\mathbb{R}}\right)$, for $n \geqslant 0$, be a sequence of uniformly bounded measurable functions with respect to a bounded Borel measure $\mu$, such that $\lim _{n \rightarrow \infty} f_{n}=f$ exists. Then $\int \lim _{n \rightarrow \infty} f_{n} d \mu=\lim _{n \rightarrow \infty} \int f_{n} d \mu$.

From this, we obtain what is essentially the $\omega$-continuity of the Lebesgue integral operator:

Corollary 8.6 [Monotone Convergence Theorem]. Let $f_{n} \in\left(X \rightarrow_{m} \mathbf{I} \overline{\mathbb{R}}\right)$, for $n \geqslant 0$, be an increasing sequence of measurable functions with respect to a bounded Borel measure $\mu$, with $f_{0}$ bounded. Then $\bigsqcup_{n \geqslant 0} \int f_{n} d \mu=\int\left(\bigsqcup_{n \geqslant 0} f_{n}\right) d \mu$.

Finally, from the Bounded Convergence Theorem above we obtain the interval version of Fatou's lemma.

Lemma 8.7 (Fatou's Lemma). Let $f_{n} \in\left(X \rightarrow_{\mathrm{m}} \mathbf{I} \overline{\mathbb{R}}\right)$, for $n \geqslant 0$, be a sequence of uniformly bounded measurable functions with respect to a bounded Borel measure $\mu$. Then $\int \lim ^{*} f_{n} d \mu \sqsubseteq \lim ^{*} \int f_{n} d \mu$.

\subsection{Computability of Lebesgue integral}

We now assume $X$ is an effectively given second countable compact Hausdorff space and $\mu$ is an effectively given finite Borel measure $\mu$ on it as described in Section 2. Recall that we have an effective enumeration $\left(O_{j}\right)_{j \geqslant 0}$ of a countable basis of $X$ with $X=O_{1}$ such that $\left(\mu\left(O_{j}\right)\right)_{j \geqslant 0}$ is a computable sequence of real numbers. The following theorem, which is our main result, brings together and uses all the results in the previous sections, on $\mu$-computable measurable sets and functions and on the interval-valued Lebesgue integral.

Theorem 8.8. Suppose $f$ is a bounded $\mu$-computable real-valued measurable function on $X$. Then the Lebesgue integral of $f$ with respect to $\mu$ is computable, i.e., given any positive integer $k$ we can effectively compute the Lebesgue integral of $f$ up to $1 / 2^{k}$ accuracy.

Proof. Let $M$ be an effectively given bound for $f$ and $\left(s_{n}\right)_{n \geqslant 0}$ be the increasing sequence of $\mu$-computable simple functions in $\left(X \rightarrow_{m} \mathbf{I} \overline{\mathbb{R}}\right)$ which witnesses the computability of $f$ according to Definition 7.7 with effectively given constant $c>0$. By the interval version of the Monotone Convergence Theorem (Corollary 8.6), we know that $\int f d \mu=\bigsqcup_{n \geqslant 0} \int s_{n} d \mu$, which means that the required integral lies in each compact interval of the shrinking sequence of compact intervals given by the integrals of the simple functions. Our task is to effectively find $n$ such that $\int s_{n} d \mu$ provides the required estimate. In fact, using the 
canonical representation:

$$
s_{n}=\sum_{i=1}^{o\left(s_{n}\right)} \alpha_{i} \chi_{A_{i}}
$$

we obtain $\int s_{n} d \mu=\sum_{i=1}^{o\left(S_{n}\right)} \alpha_{i} \mu\left(A_{i}\right)$. Since each $A_{i}$ is a $\mu$-computable measurable subset, we can effectively obtain for each nonnegative integer $p$ an open set $O_{i p}$ and a closed set $C_{i p}$ such that $C_{i p} \subseteq A_{i} \subseteq O_{i p}$ with $\mu\left(O_{i p}\right)$ and $\mu\left(C_{i p}\right)$ computable real numbers satisfying $\mu\left(O_{i p}\right)-\mu\left(C_{i p}\right)<1 / 2^{p}$.

We note that there are three types of intervals $\alpha_{i}$ in the simple map $s_{n}$ of Eq. 6: (i) $0 \leqslant \alpha_{i}^{-}$, (ii) $\alpha_{i}^{+} \leqslant 0$ and (iii) $\alpha_{i}^{-}<0<\alpha_{i}^{+}$. This gives us three pairwise disjoint subsets:

$$
\begin{aligned}
& I_{n}^{+}=\left\{0 \leqslant i \leqslant o\left(s_{n}\right): 0 \leqslant \alpha_{i}^{-}\right\} \\
& I_{n}^{-}=\left\{0 \leqslant i \leqslant 0\left(s_{n}\right): \alpha_{i}^{+} \leqslant 0\right\} \\
& I_{n}^{0}=\left\{0 \leqslant i \leqslant 0\left(s_{n}\right): \alpha_{i}^{-}<0<\alpha_{i}^{+}\right\},
\end{aligned}
$$

with $0 \leqslant i \leqslant o\left(s_{n}\right)$ iff $i \in I_{n}^{+} \cup I_{n}^{-} \cup I_{n}^{0}$.

We have

$$
\sum_{i \in I_{n}^{+}} \alpha_{i}^{-} \mu\left(C_{i p}\right)+\sum_{i \in I_{n}^{-}} \alpha_{i}^{-} \mu\left(O_{i p}\right)+\sum_{i \in I_{n}^{0}} \alpha_{i}^{-} \mu\left(O_{i p}\right) \leqslant \int f_{n} d \mu \leqslant \sum_{i \in I_{n}^{+}} \alpha_{i}^{+} \mu\left(O_{i p}\right)+\sum_{i \in I_{n}^{-}} \alpha_{i}^{+} \mu\left(C_{i p}\right)+\sum_{i \in I_{n}^{0}} \alpha_{i}^{+} \mu\left(O_{i p}\right) .
$$

Estimating the difference between the three types of terms in the two sums on the left and right hand side above we have:

$$
\begin{aligned}
\sum_{i \in I_{n}^{+}} \alpha_{i}^{+} \mu\left(O_{i p}\right)-\alpha_{i}^{-} \mu\left(C_{i p}\right) & =\sum_{i \in I_{n}^{+}} \alpha_{i}^{+} \mu\left(O_{i p}\right)-\alpha_{i}^{+} \mu\left(C_{i p}\right)+\alpha_{i}^{+} \mu\left(C_{i p}\right)-\alpha_{i}^{-} \mu\left(C_{i p}\right) \\
& =\sum_{i=1}^{o\left(S_{n}\right)} \alpha_{i}^{+}\left(\mu\left(O_{i p}\right)-\mu\left(C_{i p}\right)\right)+\left(\alpha_{i}^{+}-\alpha_{i}^{-}\right) \mu\left(C_{i p}\right) \\
& \leqslant \sum_{i \in I_{n}^{+}} \frac{m\left(S_{n}\right)}{2^{p}}+w\left(S_{n}\right) \mu\left(C_{i p}\right) \leqslant \frac{m\left(S_{n}\right) o\left(S_{n}\right)}{2^{p}}+\frac{\mu(X)}{2^{n}},
\end{aligned}
$$

since $w\left(s_{n}\right)$ is bounded by $1 / 2^{n}$ and $C_{i p}$ 's, being contained in the disjoint sets $A_{i}$, are disjoint for fixed $p$ and $1 \leqslant i \leqslant o\left(s_{n}\right)$ and their total $\mu$-measure is therefore bounded by $\mu(X)$. Thus, for our estimate, we conclude that

$$
\sum_{i \in I_{n}^{+}} \alpha_{i}^{+} \mu\left(O_{i p}\right)-\alpha_{i}^{-} \mu\left(C_{i p}\right) \leqslant \frac{m\left(s_{n}\right) c 2^{n}}{2^{p}}+\frac{\mu(X)}{2^{n}},
$$

since $o\left(s_{n}\right)$ is bounded by $c 2^{n}$.

Similary,

$$
\begin{aligned}
\sum_{i \in I_{n}^{-}} \alpha_{i}^{+} \mu\left(C_{i p}\right)-\alpha_{i}^{-} \mu\left(O_{i p}\right) & =\sum_{i \in I_{n}^{-}} \alpha_{i}^{+} \mu\left(C_{i p}\right)-\alpha_{i}^{-} \mu\left(C_{i p}\right)+\alpha_{i}^{-} \mu\left(C_{i p}\right)-\alpha_{i}^{-} \mu\left(O_{i p}\right) \\
& =\sum_{i \in I_{n}^{-}}\left(\alpha_{i}^{+}-\alpha_{i}^{-}\right) \mu\left(C_{i p}\right)+\left(\mu\left(C_{i p}\right)-\mu\left(O_{i p}\right)\right) \alpha_{i}^{-} \\
& \leqslant \frac{m\left(S_{n}\right) c 2^{n}}{2^{p}}+\frac{\mu(X)}{2^{n}},
\end{aligned}
$$

as in the computation of the estimate for the $I_{n}^{+}$terms.

Finally, we have:

$$
\begin{aligned}
\sum_{i \in I_{n}^{0}} \alpha_{i}^{+} \mu\left(O_{i p}\right)-\alpha_{i}^{-} \mu\left(O_{i p}\right) & =\sum_{i \in I_{0}^{+}}\left(\alpha_{i}^{+}-\alpha_{i}^{-}\right) \mu\left(O_{i p}\right) \\
& \leqslant \sum_{i \in I_{0}^{+}} w\left(S_{n}\right) \mu\left(O_{i p}\right) \\
& \leqslant w\left(S_{n}\right) \sum_{i \in I_{0}^{+}}\left(\mu\left(C_{i p}\right)+\frac{1}{2^{p}}\right) \leqslant \frac{\mu(X)}{2^{n}}+\frac{c}{2^{p}}
\end{aligned}
$$

Overall, collecting the contributions from the three types, we have:

$$
w\left(\int s_{n} d \mu\right) \leqslant \frac{3 \mu(X)}{2^{n}}+\frac{c\left(1+m\left(s_{n}\right) 2^{n+1}\right)}{2^{p}} .
$$

Note that $\mu(X)$ is a computable number as $X=O_{1}$; we can thus effectively obtain a nonnegative integer $t$ such that $3 \mu(X)<2^{t}$. Let the positive integer $k$ be given and put $n=k+t+1$ and

$$
p=\left\lceil\log c\left(m\left(s_{k+t+1}\right) 2^{k+t+1}+1\right) 2^{k}\right\rceil .
$$


Using the above effectively obtained $n$ and $p$, we get

$$
w\left(\int s_{n} d \mu\right) \leqslant 1 / 2^{k+1}+1 / 2^{k+1}=1 / 2^{k} .
$$

It follows that for the above values of $n$ and $p$ the computable real number

$$
\sum_{i \in I_{n}^{+}} \alpha_{i}^{+} \mu\left(O_{i p}\right)+\sum_{i \in I_{n}^{-}} \alpha_{i}^{+} \mu\left(C_{i p}\right)+\sum_{i \in I_{n}^{0}} \alpha_{i}^{+} \mu\left(O_{i p}\right)
$$

is within $1 / 2^{k}$ of the value of the integral $\int f d \mu$.

Theorem 8.8 also holds for $\mu$-recursive measurable functions which is a more general result; the proof is only slightly different and is skipped here.

\section{Conclusion and further work}

We have established a domain-theoretic computable framework for Lebesgue's measure and integration theory on locally compact Hausdorff spaces, which can herald applications of domain theory in probability theory and in the theory of $L^{p}$ spaces and functional analysis in general.

Our computability theory is based either on the extension of S̃anin's notion of $\mu$-recursive measurable sets or on the new and stronger notion of $\mu$-computable measurable sets, which gives rise to a domain-theoretic data-type for measurable sets. The classical results of Lebesgue theory are extended to interval-valued functions using the notion of interval-valued simple functions and it is shown, in particular, that the Lebesgue integral operator is $\omega$-continuous on the space of intervalvalued bounded measurable functions and that the Lebesgue integral of any $\mu$-computable real-valued bounded measurable function with respect to an effectively given finite Borel measure on an effectively given second countable compact Hausdorff space is computable. Further work is required to extend these results to the Lebesgue integral of unbounded measurable functions with respect to finite or locally finite measures on locally compact Hausdorff spaces.

One can also incorporate into this framework the idea of approximating Borel measures on second countable locally compact Hausdorff spaces by simple valuations on the upper space of the locally compact space as developed in $[9,8]$. In other words, both simple functions and simple valuations would be used to compute the Lebesgue integral. This would make the Lebesgue integral operator continuous on the product of the space of interval-valued measurable functions and the space of continuous valuations on the upper space.

\section{References}

[1] M. Alvarez-Manilla, A. Edalat, N. Saheb-Djahromi, An extension result for continuous valuations, Journal of London Mathematical Society 61 (2) (2000) 629-640.

[2] A. Bensoussan, J.L. Menadi, Stochastic hybrid control, Journal of Mathematical Analysis and Applications, 249 (2000) 261-268.

[3] J.L. Berggren, Episodes in the Mathematics of Medieval Islam, Springer, 1986.

[4] E. Bishop, D. Bridges, Constructive Analysis, Springer-Verlag, 1985.

[5] V.I. Bogachev, Measure Theory, Springer, 2007.

[6] N.J. Cutland, Computability: An Introduction to Recursive Function Theory, Cambridge University Press, 1980.

[7] J. Desharnais, A. Edalat, P. Pananagden, Bisimulation for labelled Markov processes, Information and Computation 179 (2002) $163-193$.

[8] A. Edalat, Domain theory and integration, Theoretical Computer Science 151 (1995) 163-193.

[9] A. Edalat, Dynamical systems, measures and fractals via domain theory, Information and Computation 120 (1) (1995) 32-48.

[10] A. Edalat, When Scott is weak on the top, Mathematical Structures in Computer Science 7 (1997) 401-417.

[11] A. Edalat, Semi-pullbacks and bisimulation in categories of Markov processes, Mathematical Structures in Computer Science 9 (5) (1999) 523-543.

[12] A. Edalat, R. Heckmann, A computational model for metric spaces, Theoretical Computer Science 193 (1-2)(1998) 53-73.

[13] A. Edalat, A. Lieutier, Foundation of a computable solid modelling, Theoretical Computer Science 284 (2) (2002) 319-345.

[14] A. Edalat, P. Sünderhauf, A domain theoretic approach to computability on the real line, Theoretical Computer Science 210 (1998) 73-98.

[15] P. Gaćs, Uniform test of algorithmic randomness over a general space, Theoretical Computer Science 341 (2005) 91-137.

[16] K. Ko, Complexity Theory of Real Numbers, Birkhäuser, 1991.

[17] A.N. Kolmogorov, S.V. Fomin, Introductory Real Analysis, Dover, 1975.

[18] D. Kozen, Semantics of probabilistic programs, Journal of Computer and System Sciences 22 (1981) 328-350.

[19] J.D. Lawson, Spaces of maximal points, Mathematical Structures in Computer Science 7 (5) (1997) 543-555.

[20] J.D. Lawson, B. Lu, Riemann and Edalat integration on domains, Theoretical Computer Science 305 (1-3) (2003) 259-275.

[21] Yu.I. Manin, A Course in Mathematical Logic, Springer, 1974.

[22] M.B. Pour-El, J.I. Richards, Computability in Analysis and Physics, Springer-Verlag, 1988.

[23] W. Rudin, Real and Complex Analysis, McGraw-Hill, 1970.

[24] N. Saheb-Djahromi, CPO's of measures for non-determinism, Theoretical Computer Science 12 (1) (1980) 19-37.

[25] N. S̃anin, Constructive Real Numbers and Function Spaces (E. Mendelson, Trans.), Translations of Mathematical Monographs, vol. 21, AMS, Providence Rhode Island, 1968.

[26] K. Weihrauch, Computability on the probability measures on the Borel sets of the unit interval, Theoretical Computer Science 219 (1999) $421-437$.

[27] K. Weihrauch, Computable Analysis (An Introduction), Springer, 2000.

[28] Y. Wu, D. Ding, Computability of measurable sets via effective topologies, Archive for Mathematical Logic 45 (3) (2006) 365-379.

[29] Y. Wu, K. Weihrauch, A computable version of the daniell-stone theorem on integration and linear functionals, Theoretical Computer Science 359 (1-3) (2006) 28-42. 